\title{
Altruism and Its Impact on the Price of Anarchy
}

\author{
PO-AN CHEN, National Chiao Tung University \\ BART DE KEIJZER, CWI Amsterdam \\ DAVID KEMPE, University of Southern California \\ GUIDO SCHÄFER, CWI Amsterdam and VU University Amsterdam
}

\begin{abstract}
We study the inefficiency of equilibria for congestion games when players are (partially) altruistic. We model altruistic behavior by assuming that player $i$ 's perceived cost is a convex combination of $1-\alpha_{i}$ times his direct cost and $\alpha_{i}$ times the social cost. Tuning the parameters $\alpha_{i}$ allows smooth interpolation between purely selfish and purely altruistic behavior. Within this framework, we study primarily altruistic extensions of (atomic and nonatomic) congestion games, but also obtain some results on fair cost-sharing games and valid utility games.

We derive (tight) bounds on the price of anarchy of these games for several solution concepts. Thereto, we suitably adapt the smoothness notion introduced by Roughgarden and show that it captures the essential properties to determine the robust price of anarchy of these games. Our bounds show that for atomic congestion games and cost-sharing games, the robust price of anarchy gets worse with increasing altruism, while for valid utility games, it remains constant and is not affected by altruism.

However, the increase in the price of anarchy is not a universal phenomenon: For general nonatomic congestion games with uniform altruism, the price of anarchy improves with increasing altruism. For atomic and nonatomic symmetric singleton congestion games, we derive bounds on the pure price of anarchy that improve as the average level of altruism increases. (For atomic games, we only derive such bounds when cost functions are linear.) Since the bounds are also strictly lower than the robust price of anarchy, these games exhibit natural examples in which pure Nash equilibria are more efficient than more permissive notions of equilibrium.
\end{abstract}

Categories and Subject Descriptors: F.0 [Theory of Computation]: General

General Terms: Economics, Theory

Additional Key Words and Phrases: Altruism, selfishness, congestion games, cost-sharing games, valid utility games, price of anarchy

\section{ACM Reference Format:}

Po-An Chen, Bart de Keijzer, David Kempe, and Guido Schäfer. 2014. Altruism and its impact on the price of anarchy. ACM Trans. Econom. Comput. 2, 4, Article 17 (October 2014), 45 pages.

DOI: http://dx.doi.org/10.1145/2597893

\begin{abstract}
Most of the results in this article have appeared in preliminary form in Proceedings of $7^{\text {th }}$ Workshop on Internet \& Network Economics (WINE'11) [Chen et al. 2011] and in Proceedings of the $9^{\text {th }}$ ACM Conference on Electronic Commerce (EC'08) [Chen and Kempe 2008].

Author's addresses: P.-A. Chen, Institute of Information Management, National Chiao Tung University, Taiwan; email: poanchen@nctu.edu.tw; B. de Keijzer, Networks and Optimization Group, CWI Amsterdam, The Netherlands; email: b.de.keijzer@cwi.nl; D. Kempe, Department of Computer Science, University of Southern California, USA; email: dkempe@usc.edu; G. Schäfer, Networks and Optimization Group, CWI Amsterdam and Department for Econometrics and Operations Research, VU University Amsterdam, The Netherlands; email: g.schaefer@cwi.nl.

Permission to make digital or hard copies of all or part of this work for personal or classroom use is granted without fee provided that copies are not made or distributed for profit or commercial advantage and that copies bear this notice and the full citation on the first page. Copyrights for components of this work owned by others than ACM must be honored. Abstracting with credit is permitted. To copy otherwise, or republish, to post on servers or to redistribute to lists, requires prior specific permission and/or a fee. Request permissions from permissions@acm.org.
\end{abstract}

(c) 2014 ACM $2167-8375 / 2014 / 10-A R T 17 \$ 15.00$

DOI: http://dx.doi.org/10.1145/2597893 


\section{INTRODUCTION}

Many large-scale decentralized systems, such as infrastructure investments or traffic on roads or computer networks, bring together large numbers of individuals with different and oftentimes competing objectives. When these individuals choose actions to benefit themselves, the result is frequently suboptimal for society as a whole. This basic insight has led to a study of such systems from the viewpoint of game theory, focusing on the inefficiency of stable outcomes. Traditionally, "stable outcomes" have been associated with pure Nash equilibria of the corresponding game. The notions of price of anarchy [Koutsoupias and Papadimitriou 1999] and price of stability [Anshelevich et al. 2004] provide natural measures of the system degradation, by capturing the degradation of the worst and best Nash equilibria, respectively, compared to the socially optimal outcome. However, the predictive power of such bounds has been questioned on (at least) two grounds.

(1) The assumption that players seek only to maximize their own utility is at odds with altruistic behavior routinely observed in the real world, and predicted by widely accepted evolutionary models. While modeling human incentives and behavior accurately is a formidable task, several papers have proposed natural simplified models of altruism [Ledyard 1997; Levine 1998].

(2) The adoption of Nash equilibria as a prescriptive solution concept implicitly assumes that players are able to reach such equilibria. In particular in light of several known hardness results for finding Nash equilibria, this assumption is very suspect for computationally bounded players. In response, recent work has begun analyzing the outcomes of natural response dynamics [Blum et al. 2006, 2008; Roughgarden 2009], as well as other permissive solution concepts such as correlated or coarse correlated equilibria [Aumann 1974; Hannan 1957; Roughgarden and Schoppmann 2011]. This general direction of inquiry has become known as "robust price of anarchy."

The goal of this article is to begin a thorough investigation of the effects of relaxing both of these assumptions. That is, we consider the (relaxed) equilibria reached by individuals whose utility functions contain a component of social welfare. We aim to quantify the worst-case (and in some cases, best-case) inefficiency of such equilibria. More concretely, we study the following classes of games (all of which are defined formally in Section 2).

Congestion Games. In congestion games [Roughgarden 2005; Roughgarden and Tardos 2000], there is a set of facilities, each equipped with a nondecreasing cost (or delay) function. These facilities could be roads in a road network, links in a computer network, or computational resources in a system. All of these resources become slower as the load on them increases. Individuals have strategy spaces consisting of subsets of resources, such as paths in a network, or sets of machines which can together finish a computational task. Among the different feasible sets, they will choose one to optimize their individual objective function. As a result, selfish choices can lead to overcongestion of resources that would be much faster if used in moderation.

Congestion games naturally fall into two classes: atomic and nonatomic. In atomic congestion games, individuals have nonnegligible size: even one individual will affect the cost of a facility perceptibly by using vs. not using it. In nonatomic congestion games, individuals are infinitesimally small, and there is a continuum of them. Thus, any one individual will not affect the perceived cost of a facility, and it is only the accumulation of a set of positive measure that will affect the facility's cost. 
Fair Cost-Sharing Games. Fair cost-sharing games [Anshelevich et al. 2004] also have a set of facilities, but instead of increasing the cost, the selection of a facility by many individuals will actually decrease it for each of the individuals using it. The reason is that here, selecting a facility means contributing towards its purchase: the (fixed) cost of each facility is split among all individuals contributing to it. Thus, cost-sharing games naturally model private investment in infrastructure where overall lower cost could possibly be obtained by maximizing overlap of purchased facilities. While fair cost-sharing games are thus also congestion games, their objective function behaves very differently, so that we treat them separately here.

Valid Utility Games. Valid utility games [Vetta 2002] also have individuals selecting sets of facilities, but instead of minimizing costs, individuals seek to maximize the utility they individually derive from the set of facilities selected by all players together. The social welfare also depends on the entire set of facilities selected by all individuals together. Thus, valid utility games naturally model investments in infrastructure where society loses some welfare by possible duplication between individuals' choices.

We are interested in the outcomes of these games reached by partially altruistic players. In Section 3, we formally define the altruistic extension for finite (atomic) games and for nonatomic congestion games. Our general definition is applicable to other classes of games, and is our main modeling contribution. The model is based on a suggestion of Ledyard [1997, p. 154]. In the version for atomic games, each player $i$ has an associated altruism parameter $\alpha_{i}$, and player $i$ 's cost (or payoff) is a convex combination of $\left(1-\alpha_{i}\right)$ times his direct cost (or payoff) and $\alpha_{i}$ times the social cost (or social welfare). By tuning the parameters $\alpha_{i}$, this model allows smooth interpolation between pure selfishness $\left(\alpha_{i}=0\right)$ and pure altruism $\left(\alpha_{i}=1\right)$. For non-atomic games, we argue (also in Section 3 ) that the natural analogue is to consider the derivative of the social cost or social welfare.

As discussed previously, the second modeling contribution of our work is to consider more permissive solution concepts than pure Nash equilibria, allowing us to study the joint effects of relaxing both standard assumptions. Most of our results are valid for coarse correlated equilibria and limits of best-response dynamics (defined in Section 2), using an extension of the smoothness technique of Roughgarden [2009], which constitutes the second modeling contribution of the present work. (Some results in our work only hold for pure Nash equilibria, and delineate quantitative differences between the different solution concepts.)

\subsection{Our Contributions and Results}

As discussed previously, our first main contribution is in terms of modeling: we propose a clean model of altruism that applies to most games, and extend Roughgarden's model of smoothness to it (Section 3). We provide several general results that will be useful to analyze the robust price of anarchy for games with altruism in our and other scenarios (Section 3.3). We then investigate congestion games with altruism in depth, and also show how to apply the general methodology to other games.

(1) For atomic congestion games with linear cost functions, Caragiannis et al. [2010] derived a tight bound of $\frac{5+4 \alpha}{2+\alpha}$ on the pure price of anarchy when all players have the same altruism level $\alpha{ }^{1}$ Our general framework makes it an easy observation that

\footnotetext{
${ }^{1}$ The altruism model of Caragiannis et al. [2010] differs from ours in a slight technicality discussed in Section 3 (see Remark 3.2). Therefore, various bounds we cite here are stated differently in Caragiannis et al. [2010].
} 
their proof in fact bounds the robust price of anarchy. We generalize their bound to the case when different players have different altruism levels, obtaining a bound in terms of the maximum and minimum altruism levels. (These results are presented in Section 4.)

For the special case of symmetric singleton congestion games with linear cost functions (which corresponds to selfish scheduling on machines), we can analyze some cases with nonuniform altruism. When an $\bar{\alpha}$ fraction of the players are entirely altruistic and the remaining players are entirely selfish, we obtain an improved bound of $\frac{4-2 \bar{\alpha}}{3-\bar{\alpha}}$ on the pure price of anarchy. (These bounds are proven in Section 5.)

(2) For nonatomic congestion games, we are able to bound the robust price of anarchy for any given class of cost functions on resources in terms of an optimization problem for those functions. When cost functions can be arbitrary semiconvex functions, we show that if all players are (at least) $\alpha$-altruistic, then the robust price of anarchy is always bounded by $\frac{1}{\alpha}$. For the special case of linear cost functions, our general bound implies a robust price of anarchy of $\frac{4}{3+2 \alpha-\alpha^{2}}$. (These results are the subject of Section 6.)

We also study the special case of symmetric singleton congestion games with arbitrary altruism distributions. We give a theorem characterizing the pure price of anarchy for any given class of cost functions and arbitrary altruism distributions. When the cost functions are semiconvex, the theorem implies a bound of $\frac{1}{\bar{\alpha}}$ on the pure price of anarchy, where $\bar{\alpha}$ is the mean of the altruism distribution. (These bounds are proven in Section 7.)

(3) For fair cost-sharing games, our framework lets us easily derive a bound of $\frac{n}{1-\hat{\alpha}}$ on the robust price of anarchy, where $\hat{\alpha}$ is the maximum altruism level of a player. This bound is tight for uniformly altruistic players. (This result is shown in Section 8.)

(4) For valid utility games, using our framework, we prove a tight bound of 2 on the robust price of anarchy. The bound remains at 2 regardless of the (possibly different) altruism levels of the players. (The proof is given in Section 9.)

(5) We study the general properties of the robust price of anarchy for abitrary classes of games. We prove that the robust price of anarchy behaves in a quasiconvex way. As a consequence, the worst-case robust price of anarchy is achieved at a $\{0,1\}$ altruism vector. Moreover, the set of altruism parameters for which the best price of anarchy is achieved is convex. (This is proved in Section 10.)

Notice that many of our bounds on the robust price of anarchy reveal a counterintuitive trend: For utility games, the price of anarchy is independent of the level of altruism. For atomic linear congestion games and cost-sharing games, it actually increases in the altruism level (and even goes to infinity in the case of cost-sharing games). Intuitively, this phenomenon is explained by the fact that a change of strategy by player $i$ may affect many players. An altruistic player will care more about these other players than a selfish player; hence, an altruistic player accepts more states of the game as "stable." This suggests that the best stable solution can also be chosen from a larger set, and the price of stability should thus decrease. We provide some preliminary results on the price of stability which support this intuition: for linear atomic congestion games, we derive an upper bound on the price of stability which decreases as $\frac{2}{1+\alpha}$; similarly, for cost-sharing games, we establish an upper bound which decreases as $(1-\alpha) H_{n}+\alpha$.

The increase in the price of anarchy is not a universal phenomenon: for nonatomic congestion games with altruism, the price of anarchy improves with increasing altruism. This raises the interesting question of which type of system behavior one should more likely expect in reality. The answer to this question may depend on the structure 
of the game, the levels of altruism, and the equilbrium concept. We are not aware of any systematic real-world study that has investigated this phenomenon; this is not too surprising, given the incredibly high number of variables one would need to control for. At a very high level, it appears that apparently altruistic behavior, with positive outcomes for social welfare, is frequently engaged in, for example, in environmentally conscious behavior of individuals.

\subsection{Altruism: A Discussion}

In this work, we adopt a fairly simple linear model of altruistic behavior in games. To the best of our knowledge, the first published suggestion of a similar model is due to Ledyard [1997]. Of course, a simple linear model should not be expected to accurately capture the behavior of rational agents; instead, our modeling goal is to derive qualitative insights regarding whether and what kind of impact altruism will have on the outcome of games.

That said, the type of linear expression we use is supported by widely accepted models in evolutionary biology. Hamilton's Rule [Hamilton 1963, 1964] (see also [West et al. 2011]) states the following: Let $A, B$ be two agents, and $r \in[0,1]$ a measure of how related they are, for example, in terms of genetic overlap. Consider some action, which has a cost of $c_{A}$ for $A$ and will provide a benefit of $u_{B}$ to $B$. Hamilton's rule states that we would expect for $A$ to undertake the action if $r \cdot u_{B}>c_{A}$. Indeed, as is well known among evolutionary biologists, behavior that appears altruistic (and thus irrational) is frequently explained as rational and selfish if the agents are taken to be the genes rather than the phenotypes. When the relatedness between $A$ and $B$ is not readily apparent to individual $A$ in the above example, then the level of altruism we would expect to see would correspond to the average relatedness in the population. ${ }^{2}$ Indeed, as explained by West et al. [2011] in detail, many alternative behavioral or economic models explaining altruism reduce to Hamilton's rule once relatedness uncertainty and population mixing are taken into account.

Besides models based on linear combinations of individual players' costs (as well as social welfare), several other approaches have been studied. Generally, altruism or other "other-regarding" social behavior has received attention in the behavioral economics literature (e.g., Gintis et al. [2005]). Fehr and Schmidt [2005] summarize theoretical models of other-regarding preferences that attempt to explain observed experimental evidence in different experiments. There are three main different approaches:

-Models of "social preferences" assume that a player's preference does not only depend on his own material payoff, but may also be a function of the allocation of resources to other players. Some simpler models such as the one proposed by Ledyard [1997] as well as ours fall into this category.

-Models of "interdependent preferences" assume that players care about another player's "type." For example, suppose that each player may be either of selfish type or conditionally altruistic type. If an altruistic player is aware that he interacts with another altruistic player, his preference becomes altruistic, and he is willing to be generous; if he knows that he interacts with a selfish player, his preference is to act selfishly. Some concrete examples of models in this approach include the one proposed by Levine [1998].

\footnotetext{
${ }^{2}$ At a level of individuals, or even larger groups, irrational altruistic behavior can of course arise from misestimating the relatedness $r$, or from following simple behavioral rules derived for certain relatedness values.
} 
-Models of "intention-based reciprocity" assume that players are concerned with other players' intentions. If a player perceives that the other player intends to treat him kindly, he will try to reciprocate and be kind to the other player, too; if he feels that the other player has hostile intentions, he will want to hurt that player. Thus, a player's interpretation of the other player's behavior matters crucially in this modeling approach. Alternative models such as the ones proposed by Rabin [1993] and by Geanakoplos et al. [1989] belong to this category and are designed more with the goal of modeling the psychological processes underlying spite or altruism (and reciprocity): they involve players forming beliefs about other players.

As a result, most models in the second or third approach are well suited for experimental work, but perhaps not as directly suited for the type of analysis in this article. They try to explain actual human behavior and the outcomes of economic experiments. To the best of our knowledge, our focus in this article on the effects of other-regarding behavior on social welfare has not appeared in the behavioral economics literature. A discussion of some of their advantages and disadvantages can also be found in the survey by West et al. [2011]. Distinguishing altruism, reciprocity, inequality aversion and other types of other-regarding behavior from experimental evidence can be challenging. For a discussion of experimental designs and results partly disambiguating motivations, see the classic paper by Charness and Rabin [2002].

The model of altruism used in our work can be naturally extended to include $\alpha_{i}<0$, modeling spiteful behavior. While the modeling extension is natural, many results in this and other papers do not continue to hold directly for negative $\alpha_{i}$.

\subsection{Taxes and Stackelberg Strategies}

Our definition of partial altruism naturally relates to two strategies that have been proposed in the literature for dealing with the selfishness of players: Pigou taxes and Stackelberg strategies.

The idea of taxes or tolls from a mechanism design viewpoint is to discourage players from choosing actions that will hurt social welfare. In a sense, this socializes the cost of a strategy profile. The underlying assumption is that money (taxes, tolls) and cost in the game (e.g., delay for congestion games) can be measured on the same scale, or that one can be converted linearly to the other at a player-specific rate $\rho_{i}$. Then, players will minimize a (weighted) sum of the two types of cost. If we think of charging each player the full social cost (possibly minus a large constant term), then our altruistic model captures the incentives of players by setting $\alpha_{i}=\rho_{i}$.

Consider the following toll scheme, due to Pigou [1920]: For every facility $e$, consider the load on $e$ in a socially optimal solution, and set the toll on $e$ to be the marginal cost a player would inflict on other users of $e$ by using $e$ as well. It is well known [Pigou 1920] that these tolls induce an optimal solution, in the sense that the Nash equilibrium will minimize the social cost. Our model of partial altruism, after subtracting out constant terms not under a player's control, can instead be interpreted as charging players a traffic-dependent marginal cost. Our results can thus also be interpreted as investigating the (robust) price of anarchy when different players have different tradeoffs between taxes and delay, and tolls are based on marginal costs. Similar models of tolls were considered, for example, in Dafermos [1972] and Smith [1979]. Cole et al. [2003, 2006] study optimization problems arising from nonuniform taxation in networks. (However, their goal is to minimize the total tolls, subject to forcing the flow to be optimal.)

Another interpretation of the model suggests itself when the altruism distribution has support $\{0,1\}$. In this case, a $\lambda$ fraction of players (having $\alpha_{i}=1$ ) is entirely altruistic, while a $1-\lambda$ fraction is entirely selfish. In the case of atomic games, we have seen 
that altruism can lead to worse price of anarchy, but in the case of nonatomic games, we can interpret the $\lambda$ fraction of altruistic players as being under the control of a benevolent authority aiming to minimize the social cost. When the benevolent authority commits to a strategy first, and the selfish players adapt subsequently, this is an instance of a Stackelberg game (see, e.g., Roughgarden [2004]). Indeed, any equilibrium of the game with selfish and altruistic players gives rise to a Stackelberg strategy for the benevolent authority, such that the resulting equilibrium among selfish players matches the equilibrium of the game with altruism. ${ }^{3}$ In particular, this means that all the price of anarchy bounds we derive for nonatomic congestion games with altruism support $\{0,1\}$ imply the same bounds for Stackelberg games with a benevolent authority.

\subsection{Related Work}

Much of our analysis is based on extensions of the notion of smoothness as proposed by Roughgarden [2009] (see Section 3.2). The basic idea is to bound the sum of cost increases of individual players switching strategies by a combination of the costs of two states. Because these types of bounds capture local improvement dynamics, they bound the price of anarchy not only for Nash equilibria, but also more general solution concepts, including coarse correlated equilibria. The smoothness notion was refined in the local smoothness framework by Roughgarden and Schoppmann [2011]. They require the types of bounds described above only for nearby states, thus obtaining tighter bounds, albeit only for more restrictive solution concepts and convex strategy sets. Using the local smoothness framework, they obtained optimal upper bounds for atomic splittable congestion games. Nadav and Roughgarden [2010] showed that smoothness bounds apply all the way to a solution concept called "average coarse correlated equilibrium," but not beyond.

A comparison between the costs in worst-case outcomes among sets of solution concepts was recently undertaken by Bradonjic et al. [2009] under the name "price of mediation:" Specifically, for the case of symmetric singleton congestion games with convex delay functions, they showed that the ratio between the most expensive correlated equilibrium and the most expensive Nash equilibrium can grow exponentially in the number of players.

Hayrapetyan et al. [2006] studied the impact of "collusion" in network congestion games, where players form coalitions to minimize their collective cost. These coalitions are assumed to be formed exogenously, that is, conceptually, each coalition is replaced by a "super-player" that acts on behalf of its members. The authors show that collusion in network congestion games can lead to Nash equilibria that are inferior to the ones of the collusion-free game (in terms of social cost). They also derive bounds on the price of anarchy caused by collusion. Note that the cooperation within each coalition can be interpreted as a kind of "locally" altruistic behavior, that is, each player only cares about the cost of the members of his coalition. In a sense, the setting considered in Hayrapetyan et al. [2006] can therefore be regarded as being orthogonal to the viewpoint that we adopt in this article: in their setting, players are assumed to be entirely altruistic but locally attached to their coalitions. In contrast, in our setting, players may have different levels of altruism but locality does not play a role.

Several recent studies investigate "irrational" player behavior in games; examples include studies on malicious (or spiteful) behavior [Babaioff et al. 2009; Brandt et al. 2007; Karakostas and Viglas 2007] and unpredictable (or Byzantine) behavior [Blum et al. 2008; Moscibroda et al. 2006; Roth 2008]. The work that is most related to our

\footnotetext{
${ }^{3}$ We do not know if the converse is true, that is, if every Stackelberg strategy gives rise to a Nash equilibrium of the same social cost for the game with altruism support $\{0,1\}$.
} 
work in this context is the one by Blum et al. [2008]. The authors consider repeated games in which every player is assumed to minimize his own regret. They derive bounds on the inefficiency, called total price of anarchy, of the resulting outcomes for certain classes of games, including congestion games and valid utility games. The exhibited bounds exactly match the respective price of anarchy and even continue to hold if only some of the players minimize their regret while the others are Byzantine. The latter result is surprising in the context of valid utility games because it means that the price of total anarchy remains at 2 , even if additional players are added to the game that behave arbitrarily.

Our findings allow us to draw an even more dramatic conclusion. Our bounds on the robust price of anarchy also extend to the total price of anarchy of the respective repeated games (see Section 3.3). As a consequence, our result for valid utility games implies that the price of total anarchy would remain at 2, even if the "Byzantine" players were to act altruistically. That is, while the result in Blum et al. [2008] suggests that arbitrary behavior does not harm the inefficiency of the final outcome, our result shows that altruistic behavior does not help.

Brandt et al. [2007] focus on deriving symmetric Bayesian Nash equilibria of first-price and second-price sealed bid auctions with uniform spite in the linear spite model. Their spite model is straightforward: There is a spite parameter $\beta \in[0,1]$, and the perceived utility of a player is equal to $(1-\beta)$ times his direct utility, and $-\beta$ times the sum of the utilities of the other players. In general, the expected revenue is shown to be increasing with growing uniform spite. They further show that the expected revenue in second-price auctions is higher than the expected revenue in first-price auctions when all agents are neither completely selfish nor completely spiteful. This says that spite is breaking the well-known revenue equivalence theorem, which states that a large class of auctions all yield the same revenue under certain conditions. They also prove that in the presence of spite, complete information makes equilibria for the first-price and second-price auctions identical, and reduces the revenue in second-price auctions (compared with the revenue in the Bayesian setting), while it increases the revenue in first-price auctions (compared with the revenue in the Bayesian setting).

For the generalization of congestion games to congestion games with "player-specific" cost functions (which subsume models of nonuniform altruism), Milchtaich [1996] proved the existence of pure Nash equilibria for singleton congestion games, and Ackermann et al. [2006] for matroid congestion games, in which the strategy space of each player is the basis of a matroid on the set of resources.

\subsection{Subsequent Work}

Since the original publication of the conference version of Chen and Kempe [2008], several other papers have studied identical or similar models of altruism applied to different types of games. Caragiannis et al. [2010] studied a nearly identical model of altruism in atomic congestion games. Several of our present results and models generalize results of Caragiannis et al. [2010], and we discuss them in more detail in the corresponding sections of this article.

If players' altruism levels in congestion games are not uniform (a special case of player-specific congestion games), then even the existence of pure Nash equilibria is not obvious. Hoefer and Skopalik [2009a] established it for several subclasses of atomic congestion games. They also show the existence of pure Nash equilibria and convergence for selfish scheduling with altruistic players using a time-sharing policy, while pure Nash equilibria may not exist under other standard coordination mechanisms [Hoefer and Skopalik 2009b].

Milchtaich [2012] uses the concept of "stability of equilibrium" to clarify that the real, material payoff in equilibrium for a group of altruistic players may be lower 
than for selfish or spiteful groups only when the equilibria involved are unstable. If they are stable, the total (or equivalently, average) payoff can only increase or remain unchanged with an increasing degree of altruism.

Elias et al. [2010] consider social awareness (a definition similar to our definition of altruism) in the context of network design games, and show that it improves the price of anarchy and other efficiency measures. The impact on system performance of altruistic behavior is also considered in communication networks, in particular mobile social and opportunistic networks [Hui et al. 2009a, 2009b].

Chen et al. [2010] study a model of altruism similar to ours in the context of a network vaccination game, in which each individual can decide whether or not to get vaccinated, and trades off a vaccination cost vs. the cost he might incur later if he becomes infected over the network. Pure Nash equilibria may not exist in this game, but Chen et al. [2010] show that under a natural opt-out dynamic, the price of anarchy is bounded by $1 / \alpha$. Meier et al. [2008] study a similar model in which altruism is replaced by "friendship," meaning that individuals only care about the utility of their neighbors in the network, not all nodes.

The idea of considering "friendship networks" (and thus nonuniform altruism across pairs of individuals), which is pursued by Meier et al. [2008] is also present in several other articles on "social games." For instance, such analyses are carried out in analyzing the price of anarchy and stability in Anshelevich et al. [2012] and Buehler et al. [2011].

As discussed in the context of the work by Brandt et al. [2007], altruism, spite or friendship are types of "externalities" in auction design, where bidders' perceived utilities are partly dependent on others' utilities. Fiat et al. [2013] study the design of externality-resistant auctions. They use a notion called "strong truthfulness" for preventing a decrease in performance as a result of externalities among the players.

Most recently, Anagnostopoulos et al. [2013] study a generalization of our altruism model considering player-specific directed altruistic behavior with social context and the price of anarchy in such altruistic extensions of games. Bilò et al. [2013] study similar models that can in some cases be considered generalizations or variations of our altruism model. Their work provides bounds on the price of anarchy under these models, for linear congestion games (among other classes of games). Moreover, Rahn and Schäfer [2013] introduce so-called social contribution games, where each player's individual cost is equal to the cost he induces on society because of his presence. They show that such games constitute useful abstractions of altruistic games when it comes to the analysis of the robust price of anarchy.

\section{PRELIMINARIES}

In this section, we formally introduce the classes of games studied in this article and define the solution concepts and inefficiency measures used subsequently.

Throughout this article, we define $[n]=\{1, \ldots, n\}$. Vectors are denoted in bold face. For a vector $\boldsymbol{x}$, we write $\boldsymbol{x}_{-i}$ for the vector with the $i$ th coordinate removed. We extend this notation to distributions over vectors: for a distribution $\xi$ over vectors $\boldsymbol{x}$, we will write $\xi_{-i}$ for the projection obtained by drawing a vector $\boldsymbol{x}$ according to $\xi$, then removing the $i$ th coordinate.

\subsection{Strategic Games: Definitions and Classes Studied}

Let $G=\left(N,\left(\Sigma_{i}\right)_{i \in N},\left(C_{i}\right)_{i \in N}\right)$ be a finite strategic game, where $N=[n]$ is the set of players, $\Sigma_{i}$ the strategy space of player $i, \Sigma=\Sigma_{1} \times \cdots \times \Sigma_{n}$, and $C_{i}: \Sigma \rightarrow \mathbb{R}$ the cost function of player $i$, mapping every strategy profile $s \in \Sigma$ to the player's direct cost. Unless stated otherwise, we assume that every player $i$ wants to minimize his individual cost function $C_{i}$. We also call such games cost-minimization games. A social 
cost function $C: \Sigma \rightarrow \mathbb{R}$ maps strategies to social costs. We require that $C$ is sumbounded, that is, $C(\boldsymbol{s}) \leq \sum_{i=1}^{n} C_{i}(\boldsymbol{s})$ for all $\boldsymbol{s} \in \Sigma .^{4}$

Payoff-maximization games $G=\left(N,\left(\Sigma_{i}\right)_{i \in N},\left(\Pi_{i}\right)_{i \in N}\right)$ are defined similarly. Here every player $i$ strives to maximize his payoff function $\Pi_{i}$. The social welfare function is denoted by $\Pi: \Sigma \rightarrow \mathbb{R}$.

2.1.1. Atomic Congestion Games. The bulk of this article focuses on (atomic and nonatomic) congestion games. In an atomic congestion game $G=\left(N, E,\left(\Sigma_{i}\right)_{i \in N},\left(d_{e}\right)_{e \in E}\right)$, there is a set $E$ of facilities. Players' strategies are subsets of facilities, $\Sigma_{i} \subseteq 2^{E}$. Each facility $e \in E$ has an associated delay function $d_{e}: \mathbb{N} \rightarrow \mathbb{R}$. For any set $S \subseteq \bar{E}$, we write $x_{S}(\boldsymbol{s})$ for the number of players using exactly $S$ as their strategy under $\boldsymbol{s}$. We extend this notation to write $x_{e}(s)$ for the number of players using facility $e$ as part of their strategy, that is, $x_{e}(s)=\left|\left\{i \in N: e \in s_{i}\right\}\right|$. When $s$ is clear from the context, we will omit it. Player $i$ 's cost is

$$
C_{i}(\boldsymbol{s})=\sum_{e \in s_{i}} d_{e}\left(x_{e}(\boldsymbol{s})\right)
$$

and the social cost is $C(\boldsymbol{s})=\sum_{i=1}^{n} C_{i}(\boldsymbol{s})$.

Symmetric singleton congestion games are an important special case of congestion games, in which $\Sigma_{i}=E$ for every $i .^{5}$ In that case, we will also sometimes call the facilities machines, as the game can be understood as each player choosing a machine on which to have his job processed, where the processing speed of a machine decreases as the load increases. We refer to these games simply as singleton congestion games below.

2.1.2. Nonatomic Congestion Games. Nonatomic congestion games are defined almost identically to atomic congestion games, except players now constitute a continuum, so the delay functions are defined on the nonnegative real numbers: $d_{e}: \mathbb{R}^{\geq 0} \rightarrow \mathbb{R}$. In the most general version, we only assume that each $d_{e}$ is continuous and nondecreasing. For most of the results on nonatomic congestion games, we will want to assume that delay functions are semiconvex: a function $d_{e}$ is semiconvex iff $x \cdot d_{e}(x)$ is a convex function. Notice that the set of facilities $E$ remains finite; in particular, there can only be a finite number of player types. More specifically, each player type is characterized by the collection of subsets $S \subseteq E$ of facilities that would constitute a feasible solution for the player. Thus, the player types can be associated with collections $\mathcal{S} \subseteq 2^{E}$, and there are at most $2^{2^{|E|}}$ types.

For each $\mathcal{S}$, let $r_{\mathcal{S}} \geq 0$ be the total rate of players with strategy set $\mathcal{S}$. Then, $r=\sum_{\mathcal{S} \subset 2^{E}} r_{\mathcal{S}}$ is the total rate of players. Thus, a nonatomic congestion game is characterized by $G=\left(E,\left(r_{\mathcal{S}}\right)_{\mathcal{S} \subseteq 2^{E}},\left(d_{e}\right)_{e \in E}\right)$. In specifying the strategy profiles, we avoid the $\boldsymbol{s}$ notation, since we have a continuum of players. Strategy profiles of non-atomic games are characterized by flows: For each type $\mathcal{S}$, we have a flow $f_{\mathcal{S}, S} \geq 0$ for each set of facilities $S \in \mathcal{S}$, such that $\sum_{S \in \mathcal{S}} f_{\mathcal{S}, S}=r_{\mathcal{S}}$. We also call the $f_{\mathcal{S}, S}$ feasible flows. Notice that while (single-commodity or multi-commodity) network flows are examples of flows for nonatomic congestion games, our definition extends to much more general settings. For notational convenience, we also write $f_{S}=\sum_{\mathcal{S} \ni S} f_{\mathcal{S}, S}$ for the overall flow on a set $S$, and $f_{e}=\sum_{S \ni e} f_{S}$ for the overall flow on facility $e$. Similar to the atomic case, we call

\footnotetext{
${ }^{4}$ The most "natural" special case is studying the social welfare, that is, the case when $C(\boldsymbol{s})=\sum_{i=1}^{n} C_{i}(\boldsymbol{s})$. However, many of our results apply to the more general setting, and some games are most naturally modeled in this framework.

${ }^{5}$ We sometimes identify singleton sets with their element to improve readability, and write $e$ instead of $\{e\}$. We will do so only when we believe that no confusion can arise, and to stress one aspect or the other of the strategy under consideration.
} 
a nonatomic congestion game a singleton congestion game if all players have the same type $\mathcal{S}$ and $\mathcal{S}=E$.

2.1.3. Cost-Sharing Games. A fair cost-sharing game $G=\left(N, E,\left(\Sigma_{i}\right)_{i \in N},\left(c_{e}\right)_{e \in E}\right)$ is defined similarly to an atomic congestion game. The difference is in the nature of the cost functions only: for a fair cost-sharing game, the individuals' costs are

$$
C_{i}(\boldsymbol{s})=\sum_{e \in s_{i}} \frac{c_{e}}{x_{e}(\boldsymbol{s})}
$$

This captures that the cost $c_{e}$ of acquiring the necessary facilities is shared evenly between all users. In particular, usage of a resource by multiple players decreases the cost to each of them, rather than increasing it. For notational convenience, we write $U(\boldsymbol{s})=\bigcup_{i \in N} s_{i}$ for the set of all facilities used by at least one player. The social cost function is then $C(\boldsymbol{s})=\sum_{i=1}^{n} C_{i}(\boldsymbol{s})=\sum_{e \in U(\boldsymbol{s})} c_{e}$.

2.1.4. Valid Utility Games. A valid utility game [Vetta 2002] is a payoff maximization game given by $G=\left(N, E,\left(\Sigma_{i}\right)_{i \in N},\left(\Pi_{i}\right)_{i \in N}, V\right)$. Again, $E$ is a ground set of resources, and the strategy sets $\Sigma_{i}$ are subsets of $2^{E} . \Pi_{i}$ is the payoff function that player $i$ wants to maximize, and $V$ is a submodular ${ }^{6}$ and nonnegative function on $E$. As before, let $U(\boldsymbol{s})=\bigcup_{i \in N} s_{i} \subseteq E$ be the union of all players' strategies under $\boldsymbol{s}$. The social welfare function $\Pi: \Sigma \rightarrow \mathbb{R}$ to be maximized is $\Pi(\boldsymbol{s})=V(U(\boldsymbol{s}))$, and thus depends only on the union of the players' chosen strategies, evaluated by $V$. The individual payoff functions of all players $i \in N$ are assumed to satisfy ${ }^{7} \Pi_{i}(\boldsymbol{s}) \geq \Pi(\boldsymbol{s})-\Pi\left(\varnothing, \boldsymbol{s}_{-i}\right)$ for every strategy profile $s \in \Sigma$. Intuitively, this means that the individual payoff of a player is at least his contribution to the social welfare. Moreover, it is assumed that $\Pi(\boldsymbol{s}) \geq \sum_{i=1}^{n} \Pi_{i}(\boldsymbol{s})$ for every $s \in \Sigma$. See Vetta [2002] for a detailed description and justification of these assumptions.

Examples of games falling into this framework include natural game-theoretic variants of the facility location, $k$-median and network routing problems [Vetta 2002].

\subsection{Equilibrium Concepts}

The most general equilibrium concept that we will deal with in this article is the following one.

Definition 2.1 (Coarse Equilibrium). A coarse equilibrium (or coarse correlated equilibrium) of a game $G$ is a probability distribution $\sigma$ over $\Sigma=\Sigma_{1} \times \cdots \times \Sigma_{n}$ with the following property: if $s$ is a random variable with distribution $\sigma$, then for each player $i$, and all $s_{i}^{*} \in \Sigma_{i}$ :

$$
\mathbf{E}_{\boldsymbol{s} \sim \sigma}\left[C_{i}(\boldsymbol{s})\right] \leq \mathbf{E}_{\boldsymbol{s}_{-i} \sim \sigma_{-i}}\left[C_{i}\left(s_{i}^{*}, \boldsymbol{s}_{-i}\right)\right] .
$$

The set of all coarse equilibria is also known as the Hannan Set (see, e.g., Young [1995]). It includes several other solution concepts, such as correlated equilibria, mixed Nash equilibria and pure Nash equilibria. We briefly review these equilibrium notions.

Informally, the difference between a coarse equilibrium and a correlated equilibrium is the following: in a coarse equilibrium, a player will play according to $s$ when he is informed of the distribution $\sigma$ from which $\boldsymbol{s}$ is drawn. In a correlated equilibrium, a player will play according to $s$ when he is informed of the distribution $\sigma$ as well as the strategy that has been drawn for him, that is, that he will play under s. More formally,

\footnotetext{
${ }^{6}$ For a finite set $E$, a function $f: 2^{E} \rightarrow \mathbb{R}$ is submodular iff $f(A \cup\{e\})-f(A) \geq f(B \cup\{e\})-f(B)$ for any $A \subseteq B \subseteq E, e \in E$.

${ }^{7}$ We abuse notation and write $\Pi\left(\varnothing, \boldsymbol{s}_{-i}\right):=V\left(\bigcup_{j \in N \backslash\{i\}} s_{j}\right)$.
} 
this means that in a correlated equilibrium, for all $s_{i}^{*} \in \Sigma_{i}$,

$$
\mathbf{E}_{\boldsymbol{s} \sim \sigma}\left[C_{i}(\boldsymbol{s})\right] \leq \mathbf{E}_{\boldsymbol{s} \sim \sigma}\left[C_{i}\left(s_{i}^{*}, \boldsymbol{s}_{-i}\right)\right] .
$$

A mixed Nash equilibrium is a coarse equilibrium whose distribution $\sigma$ is the Cartesian product of independent distributions $\sigma_{1}, \ldots, \sigma_{n}$ for the players. Thus, any mixed Nash equilibrium is also a correlated equilibrium. A pure Nash equilibrium is a strategy profile $\boldsymbol{s}$ such that for each player $i, C_{i}(\boldsymbol{s}) \leq C_{i}\left(s_{i}^{\prime}, \boldsymbol{s}_{-i}\right)$ for all $s_{i}^{\prime} \in \Sigma_{i}$. A pure Nash equilibrium is a special case of a mixed Nash equilibrium where the support of $\sigma_{i}$ has cardinality 1 for all $i$.

We use $\operatorname{PNE}(G), \operatorname{MNE}(G), \operatorname{CE}(G)$, and $\operatorname{CCE}(G)$ to denote the set of pure Nash equilibria, mixed Nash equilibria, correlated equilibria, and coarse equilibria of a game $G$, respectively.

\subsection{Inefficiency of Equilibria}

The price of anarchy [Koutsoupias and Papadimitriou 1999] and price of stability [Anshelevich et al. 2004] are natural ways of quantifying the inefficiency of equilibria for classes of games:

Definition 2.2 (Price of Anarchy, Price of Stability). Let $S \subseteq \Sigma$ be a set of strategy profiles for a cost-minimization game $G$ with social cost function $C$, and let $\boldsymbol{s}^{*}$ be a strategy profile that minimizes $C$. We define

$$
\operatorname{PoA}(S, G)=\sup _{\boldsymbol{s} \in S} \frac{C(\boldsymbol{s})}{C\left(\boldsymbol{s}^{*}\right)} \quad \text { and } \quad \operatorname{PoS}(S, G)=\inf _{\boldsymbol{s} \in S} \frac{C(\boldsymbol{s})}{C\left(\boldsymbol{s}^{*}\right)} .
$$

The coarse (respectively, correlated, mixed, pure) price of anarchy of a class of games $\mathcal{G}$ is defined as

$$
\sup _{G \in \mathcal{G}} \operatorname{PoA}\left(S_{G}, G\right)
$$

where $S_{G}=\operatorname{CCE}(G)$ (respectively, $\operatorname{CE}(G), \operatorname{MNE}(G), \operatorname{PNE}(G)$ ). The coarse (respectively, correlated, mixed, pure) price of stability of a class of games is defined analogously, that is, by replacing PoA by PoS in Eq. (3).

We extend Definition 2.2 in the obvious way to payoff-maximization games $G$ with social welfare function $\Pi$ by considering the ratio $\Pi\left(s^{*}\right) / \Pi(s)$, where $\boldsymbol{s}^{*}$ refers to a strategy profile maximizing $\Pi$.

\section{ALTRUISM MODEL AND SMOOTHNESS TECHNIQUE}

We first introduce our altruism model and show how the smoothness approach of Roughgarden [2009] can be extended to altruistic games.

\subsection{Altruistic Extensions}

We study altruistic extensions of strategic games equipped with sum-bounded social cost functions. Our definition is based on a suggestion first put forward by Ledyard [1997] (to the best of our knowledge). We first define the altruistic extension for finite games, and then extend it to games with a continuum of players; the latter extension, however, is only defined for nonatomic congestion games.

3.1.1. Finite Games. We define altruistic extensions of finite games as follows:

Definition 3.1 (Altruistic extension of atomic games). Let $G=\left(N,\left(\Sigma_{i}\right)_{i \in N},\left(C_{i}\right)_{i \in N}\right)$ be a finite cost-minimization game with a sum-bounded social cost function $C$. Let $\alpha \in[0,1]^{n}$. The $\alpha$-altruistic extension of $G$ (or simply $\alpha$-altruistic game) is defined as 
the strategic game $G^{\alpha}=\left(N,\left(\Sigma_{i}\right)_{i \in N},\left(C_{i}^{\alpha}\right)_{i \in N}\right)$, where for every $i \in N$ and $s \in \Sigma$,

$$
C_{i}^{\alpha}(\boldsymbol{s})=\left(1-\alpha_{i}\right) C_{i}(\boldsymbol{s})+\alpha_{i} C(\boldsymbol{s}) .
$$

Thus, the perceived cost $C_{i}^{\alpha}(\boldsymbol{s})$ that player $i$ experiences under $\boldsymbol{s}$ is a convex combination of his direct (selfish) cost $C_{i}(\boldsymbol{s})$ and the social cost $C(\boldsymbol{s})$; we call such a player $\alpha_{i}$-altruistic. ${ }^{8}$ When $\alpha_{i}=0$, player $i$ is entirely selfish; thus, $\boldsymbol{\alpha}=\mathbf{0}$ recovers the original game. A player with $\alpha_{i}=1$ is entirely altruistic. Given an altruism vector $\alpha \in[0,1]^{n}$, we let $\hat{\alpha}=\max _{i \in N} \alpha_{i}$ and $\check{\alpha}=\min _{i \in N} \alpha_{i}$ denote the maximum and minimum altruism levels, respectively. When $\alpha_{i}=\alpha$ (a scalar) for all $i$, we call such games uniformly $\alpha$-altruistic games.

Remark 3.2. In a recent paper, Caragiannis et al. [2010] model uniformly altruistic players by defining the perceived cost of player $i$ as $(1-\xi) C_{i}(\boldsymbol{s})+\xi\left(C(\boldsymbol{s})-C_{i}(\boldsymbol{s})\right)$, where $\xi \in[0,1]$. It is not hard to see that in the range $\xi \in\left[0, \frac{1}{2}\right]$ this definition is equivalent to ours by setting $\alpha=\xi /(1-\xi)$ or $\xi=\alpha /(1+\alpha){ }^{9}$

The altruistic extension of a payoff-maximization game, in which players seek to maximize their payoff functions $\left(\Pi_{i}\right)_{i \in N}$, with a social welfare function $\Pi$ is defined analogously to Definition 3.1; the only difference is that every player $i$ wants to maximize $\Pi_{i}^{\alpha}$ instead of minimizing $C_{i}^{\alpha}$ here.

3.1.2. Nonatomic Congestion Games. To motivate the definition of the altruistic extension of nonatomic congestion games, we consider them as the limit of finite congestion games as the number of players grows to infinity. If we increase the number of players while keeping the "scale" of the delay functions constant, the delay on the (finitely many) facilities will grow to infinity, so in the limit, the comparison of strategies will be meaningless. Probably the most natural way to circumvent this issue is to keep the total "rate" of players constant, and make the impact of each individual player smaller as the number of players grows.

Specifically, fix a total rate $r_{\mathcal{S}}$ for each possible player type $\mathcal{S} \subseteq 2^{E}$, and let $r=$ $\sum_{\mathcal{S} \subseteq 2^{E}} r_{\mathcal{S}}$ be the total rate of players. The delay functions of the non-atomic game are $d_{e}:[0, r] \rightarrow \mathbb{R}$. When we consider an atomic game with $N$ players, we will consider having $N_{\mathcal{S}}=N \cdot \frac{r_{S}}{r}$ players of type $\mathcal{S}$, and define the cost functions $D_{e}(k):=d_{e}(r k / N)$. (Here, and subsequently, we will treat $N_{\mathcal{S}}$ and similar large numbers as integers, and omit ceiling/floor operators.) Consider a strategy profile in which a player with altruism $\alpha$ plays the strategy $S \subseteq E$; for each $e \in E$, as before, let $x_{e}$ be the number of players whose strategy includes the facility $e$. Equation (4) for this player's perceived cost then becomes

$$
C^{\alpha}(\boldsymbol{s})=(1-\alpha) \sum_{e \in S} D_{e}\left(x_{e}\right)+\alpha \sum_{e \in E} x_{e} D_{e}\left(x_{e}\right) .
$$

Define $x_{e}^{\prime}=x_{e}-1$ for $e \in S$, and $x_{e}^{\prime}=x_{e}$ for $e \notin S$ to be the total load on each resource when the player is not participating at all. Thus, $x^{\prime}$ is independent of the strategy chosen by this player, and minimizing $C^{\alpha}(\boldsymbol{s})$ is equivalent to minimizing

$$
C^{\alpha}(\boldsymbol{s})-\alpha \sum_{e \in E} x_{e}^{\prime} D_{e}\left(x_{e}^{\prime}\right)=(1-\alpha) \sum_{e \in S} D_{e}\left(x_{e}\right)+\alpha \sum_{e \in S}\left(x_{e} D_{e}\left(x_{e}\right)-\left(x_{e}-1\right) D_{e}\left(x_{e}-1\right)\right) .
$$

\footnotetext{
${ }^{8}$ We note that the altruistic part of an individual's perceived cost does not recursively take other players' perceived cost into account. Such recursive definitions of altruistic utility have been studied, for example, by Bergstrom [1999], and can be reduced to our definition under suitable technical conditions.

${ }^{9}$ The model of Caragiannis et al. [2010] with $\xi \in\left(\frac{1}{2}, 1\right]$ has players assign strictly more weight to others than to themselves, a possibility not present in our model.
} 
We express the number of players on a facility $e$ in terms of the flow on the facility as follows: $x_{e}=N f_{e} / r$. We substitute this definition as well as the definition of $D_{e}$, and then write $\delta=r / N$, to obtain that the player minimizes

$$
\begin{gathered}
(1-\alpha) \sum_{e \in S} d_{e}\left(f_{e}\right)+\alpha \sum_{e \in S} \frac{\left(f_{e} d_{e}\left(f_{e}\right)-\left(f_{e}-r / N\right) d_{e}\left(f_{e}-r / N\right)\right)}{r / N} \\
=(1-\alpha) \sum_{e \in S} d_{e}\left(f_{e}\right)+\alpha \sum_{e \in S} \frac{\left(f_{e} d_{e}\left(f_{e}\right)-\left(f_{e}-\delta\right) d_{e}\left(f_{e}-\delta\right)\right)}{\delta} .
\end{gathered}
$$

Keeping the total rate $r$ of players fixed, and letting $N \rightarrow \infty$ results in $\delta \rightarrow 0$, which means that the expression that the player minimizes converges to

$$
(1-\alpha) \sum_{e \in S} d_{e}\left(f_{e}\right)+\alpha \sum_{e \in S}\left(f_{e} d_{e}\left(f_{e}\right)\right)^{\prime}
$$

This derivation motivates the following definition.

Definition 3.3. In an altruistic extension of a nonatomic congestion game, each $\alpha$ altruistic player of type $\mathcal{S}$ with $\alpha \in[0,1]$ chooses a subset $S \in \mathcal{S}$ so as to minimize the cost function

$$
d_{S}^{(\alpha)}(f):=(1-\alpha) \sum_{e \in S} d_{e}\left(f_{e}\right)+\alpha \sum_{e \in S}\left(f_{e} d_{e}\left(f_{e}\right)\right)^{\prime} .
$$

The expression $\left(f_{e} d_{e}\left(f_{e}\right)\right)^{\prime}$ denotes the derivative with respect to $f_{e}$. Notice that we can rewrite $d_{S}^{(\alpha)}(f)=\sum_{e \in S} d_{e}\left(f_{e}\right)+\alpha \sum_{e \in S} f_{e} d_{e}^{\prime}\left(f_{e}\right)$.

While our definition is motivated mathematically, there is a "psychological" interpretation of the underlying choice: in order to behave (partially) altruistically, infinitesimally small players must give infinitesimally small weight to their own cost or payoff, which is achieved implicitly by making the altruistic component the derivative of the social welfare. When there are infinitely many infinitesimally small players, then no individual's actions will have an impact on the overall welfare. This kind of reasoning can be observed in the real world when individuals refuse to take the "right" action on the grounds that their actions "don't matter" (e.g., "It does not affect pollution whether I personally drive to work"). Altruistic behavior is more likely to arise when individuals project their actions on the population as a whole ("What would happen if everyone acted the way I do?"), which is mathematically accomplished by considering the rate of change of the welfare.

Based on Definition 3.3, we would like to define the altruistic extension of a game with possibly different altruism levels for different players. In the most general case, for each possible player type $\mathcal{S}$, we are given an arbitrary altruism density function $\psi_{\mathcal{S}}$ on the interval $[0,1]$. We only require that all these functions $\psi_{\mathcal{S}}$ be indeed distributions, that is, forming a Borel measure of total measure 1 . If the rate for type $\mathcal{S}$ is $r_{\mathcal{S}}$, then the overall altruism density function is $\psi=\frac{1}{r} \sum_{\mathcal{S}} r_{\mathcal{S}} \psi_{\mathcal{S}}$. The average altruism of a distribution $\psi$ is then $\int_{0}^{1} t \psi(t) d t$. An instance of the altruistic extension of a non-atomic congestion game is thus the quadruple $\left(E, \boldsymbol{r}, \boldsymbol{d},\left(\psi_{\mathcal{S}}\right)_{\mathcal{S} \subseteq 2^{E}}\right)$. In symmetric nonatomic congestion games, in which all players are of the same type $\mathcal{S} \subseteq 2^{E}$, we write $(E, \boldsymbol{r}, \boldsymbol{d}, \psi)$, and if the altruism is uniform (i.e., the distribution deterministically takes the value $\alpha)$, we simplify further to $(E, \boldsymbol{r}, \boldsymbol{d}, \alpha)$.

\subsection{Inefficiency of Altruistic Games and Smoothness}

Many proofs bounding the price of anarchy for specific games (e.g., Roughgarden [2005] and Vetta [2002]) use the fact that deviating from an equilibrium to the strategy at 
optimum is not beneficial for any player. The addition of these inequalities, combined with suitable properties of the social cost function, then gives a bound on the equilibrium's cost. Roughgarden [2009] captured the essence of this type of argument with his definition of $(\lambda, \mu)$-smoothness of a game, thus providing a generic template for proving bounds on the price of anarchy. Indeed, because such arguments only reason about local moves by players, they immediately imply bounds not only for Nash equilibria, but all classes of equilibria defined in Section 2.2, as well as the outcomes of no-regret sequences of play [Blum et al. 2006, 2008]. Recent work has explored both the limits of this concept [Nadav and Roughgarden 2010] and a refinement requiring smoothness only in local neighborhoods [Roughgarden and Schoppmann 2011]. The latter permits more fine-grained analysis of games, but applies only to correlated equilibria and their subclasses.

In this article, we study the price of anarchy and the price of stability of altruistic extensions $G^{\alpha}$ with respect to the original social cost function $C$, not accounting for the altruistic components. This reflects our desire to understand the overall performance of the system (or strategic game), which is not affected by different perceptions of costs by individuals. Note, however, that if all players have a uniform altruism level $\alpha_{i}=\alpha \in[0,1]$ and the social cost function $C$ is equal to the sum of all players' individual costs, then for every strategy profile $s \in \Sigma, C^{\alpha}(\boldsymbol{s})=(1-\alpha+\alpha n) C(\boldsymbol{s})$, where $C^{\alpha}(\boldsymbol{s})=$ $\sum_{i \in N} C_{i}^{\alpha}(s)$ denotes the sum of all players' perceived costs. In particular, bounding the price of anarchy with respect to $C$ is equivalent to bounding the price of anarchy with respect to total perceived $\operatorname{cost} C^{\alpha}$ in this case.

In extending the definition of smoothness to altruistic games, we have to exercise some care. Simply applying Roughgarden's definition to the new game does not work, as the social cost function we wish to bound is the sum of all direct costs without consideration of the altruistic component. Thus, with respect to the social cost, altruistic games are in general not sum-bounded. For this reason, we propose a slightly revised definition of $(\lambda, \mu, \boldsymbol{\alpha})$-smoothness.

For notational convenience, we define $C_{-i}(\boldsymbol{s})=C(\boldsymbol{s})-C_{i}(\boldsymbol{s}) \leq \sum_{j \neq i} C_{j}(\boldsymbol{s})$. Note that when the social cost is the sum of all players' costs, the inequality is an equality.

Definition $3.4((\lambda, \mu, \alpha)$-smoothness $)$. Let $G^{\alpha}$ be an $\boldsymbol{\alpha}$-altruistic extension of a game with sum-bounded social cost function $C . G^{\alpha}$ is $(\lambda, \mu, \alpha)$-smooth if for any two strategy profiles $\boldsymbol{s}, \boldsymbol{s}^{\prime} \in \Sigma$,

$$
\sum_{i=1}^{n} C_{i}\left(s_{i}^{\prime}, \boldsymbol{s}_{-i}\right)+\alpha_{i}\left(C_{-i}\left(s_{i}^{\prime}, \boldsymbol{s}_{-i}\right)-C_{-i}(\boldsymbol{s})\right) \leq \lambda C\left(\boldsymbol{s}^{\prime}\right)+\mu C(\boldsymbol{s}) .
$$

We also define the notion of smoothness for non-atomic games. To avoid notational overload, we only define it for the case of uniform altruism $\alpha$; we only use it in this case here. For a nonatomic game $G^{\alpha}=(E, \boldsymbol{r}, \boldsymbol{d}, \alpha)$, we say that $G^{\alpha}$ is $(\lambda, \mu, \alpha)$-smooth if for any two feasible flows $f$ and $f^{\prime}$,

$$
\sum_{e \in E}\left(f_{e}^{\prime} d_{e}\left(f_{e}\right)+\left(f_{e}^{\prime}-f_{e}\right) \alpha f_{e} d_{e}^{\prime}\left(f_{e}\right)\right) \leq \lambda C\left(f^{\prime}\right)+\mu C(f) .
$$

For $\boldsymbol{\alpha}=\mathbf{0}$, this definition coincides with Roughgarden's notion of $(\lambda, \mu)$-smoothness, and indeed, we recover Roughgarden's smoothness result as a special case of Proposition 3.5. To gain some intuition, consider two strategy profiles $\boldsymbol{s}, \boldsymbol{s}^{\prime} \in \Sigma$, and a player $i \in N$ who switches from his strategy $s_{i}$ under $\boldsymbol{s}$ to $s_{i}^{\prime}$, while the strategies of the other players remain fixed at $\boldsymbol{s}_{-i}$. The contribution of player $i$ to the left-hand side of (5) then accounts for the individual cost that player $i$ perceives after the switch, plus $\alpha_{i}$ times the difference in social cost caused by this switch excluding player $i$. The sum of these 
contributions needs to be bounded by $\lambda C\left(\boldsymbol{s}^{\prime}\right)+\mu C(\boldsymbol{s})$. We will see that this definition of $(\lambda, \mu, \boldsymbol{\alpha})$-smoothness allows us to quantify the price of anarchy of some large classes of altruistic games with respect to the very broad class of coarse correlated equilibria.

The definition of smoothness can be naturally extended to payoff maximization games. Let $G^{\alpha}$ be an $\alpha$-altruistic extension of a payoff maximization game with social welfare function $\Pi$. Define $\Pi_{-i}(\boldsymbol{s})=\Pi(\boldsymbol{s})-\Pi_{i}(\boldsymbol{s}) . G^{\alpha}$ is $(\lambda, \mu, \boldsymbol{\alpha})$-smooth iff for every two strategy profiles $\boldsymbol{s}, \boldsymbol{s}^{\prime} \in \Sigma$,

$$
\sum_{i=1}^{n} \Pi_{i}\left(s_{i}^{\prime}, \boldsymbol{s}_{-i}\right)+\alpha_{i}\left(\Pi_{-i}\left(s_{i}^{\prime}, \boldsymbol{s}_{-i}\right)-\Pi_{-i}(\boldsymbol{s})\right) \geq \lambda \Pi\left(\boldsymbol{s}^{\prime}\right)-\mu \Pi(\boldsymbol{s}) .
$$

\subsection{Robust Price of Anarchy}

As a useful tool for our subsequent analysis, we first show that many of the results in Roughgarden [2009] following from $(\lambda, \mu)$-smoothness carry over to our altruistic setting using the extended $(\lambda, \mu, \alpha)$-smoothness notion (Definition 3.4). Even though some care has to be taken in extending these results, most of the proofs of the propositions in this section follow along similar lines as their analogues in Roughgarden [2009]. The proofs are in Appendix A.

Proposition 3.5. Let $G^{\alpha}$ be an $\alpha$-altruistic extension of a game with sum-bounded social cost function $C$. If $G^{\alpha}$ is $(\lambda, \mu, \alpha)$-smooth with $\mu<1$, then the coarse (and thus correlated, mixed, and pure) price of anarchy of $G^{\alpha}$ is at most $\frac{\lambda}{1-\mu}$.

For $\boldsymbol{\alpha}=\mathbf{0}$, we recover Roughgarden's smoothness result as a special case. Proposition 3.5 can be naturally extended to smooth nonatomic congestion games with uniform altruism.

Proposition 3.6. Let $G^{\alpha}$ be a uniformly $\alpha$-altruistic nonatomic congestion game. If $G^{\alpha}$ is $(\lambda, \mu, \alpha)$-smooth with $\mu<1$, then the coarse price of anarchy of $G^{\alpha}$ is at most $\frac{\lambda}{1-\mu}$.

As we show later, for many important classes of games, the bounds obtained by $(\lambda, \mu, \boldsymbol{\alpha})$-smoothness arguments are actually tight, even for pure Nash equilibria. Therefore, as in Roughgarden [2009], we define the robust price of anarchy as the best possible bound on the coarse price of anarchy obtainable by a $(\lambda, \mu, \alpha)$-smoothness argument.

Definition 3.7. The robust price of anarchy of an $\alpha$-altruistic game $G^{\alpha}$ is defined as

$$
\operatorname{RPoA}_{G}(\boldsymbol{\alpha})=\inf \left\{\frac{\lambda}{1-\mu}: G^{\alpha} \text { is }(\lambda, \mu, \boldsymbol{\alpha}) \text {-smooth, } \mu<1\right\} .
$$

For a class $\mathcal{G}$ of games, we define $\operatorname{RPoA}_{\mathcal{G}}(\boldsymbol{\alpha})=\sup _{G \in \mathcal{G}} \operatorname{RPoA}_{G}(\boldsymbol{\alpha})$. We omit the subscript when the game (or class of games) is clear from the context.

The definition extends naturally to nonatomic congestion games; we will only consider such games with uniform altruism $\alpha$.

The smoothness condition also proves useful in the context of no-regret sequences and the price of total anarchy, introduced by Blum et al. [2008].

Proposition 3.8. Let $G^{\alpha}$ be an $\alpha$-altruistic extension of a game with sum-bounded social cost function $C$. Let $\boldsymbol{s}^{*}$ be a strategy profile minimizing the social cost function $C$ of $G^{\alpha}$, and let $\boldsymbol{s}^{1}, \ldots, \boldsymbol{s}^{T}$ be a sequence of strategy profiles in which every player $i \in N$ 
experiences vanishing average external regret, that is,

$$
\sum_{t=1}^{T} C_{i}^{\alpha}\left(\boldsymbol{s}^{t}\right) \leq\left(\min _{s_{i}^{\prime} \in \Sigma_{i}} \sum_{t=1}^{T} C_{i}^{\alpha}\left(s_{i}^{\prime}, \boldsymbol{s}_{-i}^{t}\right)\right)+o(T) .
$$

The average cost of this sequence of $T$ strategy profiles then satisfies

$$
\frac{1}{T} \sum_{t=1}^{T} C\left(s^{t}\right) \leq R P o A(\alpha) \cdot C\left(s^{*}\right) \quad \text { as } T \rightarrow \infty .
$$

Proposition 3.8 extends to the case of nonatomic congestion games. In order to state the conditions and proof precisely, we use the following notation. Let $\tau:[0, r] \rightarrow$ $\{\mathcal{S}: \mathcal{S}$ is a type of player $\}$ be any (fixed) map with the property that $\left|\tau^{-1}(\mathcal{S})\right|=r_{\mathcal{S}}$ for all types $\mathcal{S}$. In other words, we associate the continuum of players with the interval $[0, r]$ and associate with each point $y$ the type of the particular player. Then, we consider a valid flow $f$ as a mapping $\phi:[0, r] \rightarrow\left\{S \in 2^{E}\right\}$ with the property that $\phi(y) \in \tau(y)$; in other words, we assign a strategy to each among the continuum of players. ${ }^{10}$

Proposition 3.9. Consider a uniformly $\alpha$-altruistic nonatomic congestion game $G^{\alpha}$ that is $(\lambda, \mu, \alpha)$-smooth with $\mu<1$. Let $f^{*}$ be a flow minimizing the social cost $C$, and $f^{1}, \ldots, f^{T}$ a sequence of flows in which every infinitesimal player $y \in[0, r]$ experiences vanishing average external regret in the following sense:

$$
\sum_{t=1}^{T} \sum_{e \in \phi^{t}(y)} d_{e}\left(f_{e}^{t}\right)+\alpha f_{e}^{t} d_{e}^{\prime}\left(f_{e}^{t}\right) \leq \min _{\hat{S} \in \tau(y)} \sum_{t=1}^{T} \sum_{e \in \hat{S}}\left(d_{e}\left(f_{e}^{t}\right)+\alpha f_{e}^{t} d_{e}^{\prime}\left(f_{e}^{t}\right)\right)+o(T) .
$$

The average cost of this sequence of $T$ flows then satisfies

$$
\frac{1}{T} \sum_{t=1}^{T} C\left(f^{t}\right) \leq R P o A(\alpha) \cdot C\left(f^{*}\right)+o(1)
$$

as $T \rightarrow \infty$.

The results in this section continue to hold for altruistic extensions of payoffmaximization games using the modified definition according to (7). Given this smoothness definition, all these results hold when we replace $\frac{\lambda}{1-\mu}$ by $\frac{1+\mu}{\lambda}$ and $\mu<1$ by $\mu>-1$ in Definition 3.7.

\section{ATOMIC CONGESTION GAMES}

We begin by studying atomic linear congestion games. Atomic congestion games were defined in Section 2.1.1; linear congestion games are the special case in which the delay functions are of the form $d_{e}(x)=a_{e} x+b_{e}$, where $a_{e}, b_{e}$ are non-negative rational numbers. Pure Nash equilibria of altruistic extensions of linear congestion games always exist [Hoefer and Skopalik 2009a]; this may not be the case for arbitrary (nonlinear) congestion games.

The price of anarchy of linear congestion games (without altruism) is known to be $\frac{5}{2}$ [Christodoulou and Koutsoupias 2005]. Recently, Caragiannis et al. [2010] extended this result to linear congestion games with uniformly altruistic players. Applying the transformation outlined in Remark 3.2, their result can be stated as follows:

${ }^{10}$ This assignment is of course not unique, and we choose one arbitrarily. 
TheOREM 4.1 (CARAgIANNIS ET AL. [2010]). The pure price of anarchy of uniformly $\alpha$ altruistic linear congestion games is at most $\frac{5+4 \alpha}{2+\alpha}$.

The proof in Caragiannis et al. [2010] implicitly uses a smoothness argument in the framework we define here for altruistic games. Thus, without any additional work, our framework allows the extension of Theorem 4.1 to the robust PoA. Caragiannis et al. [2010] also showed that the bound of Theorem 4.1 is asymptotically tight, based on singleton examples. A somewhat simpler nonsingleton example (given here) proves tightness of this bound (not only asymptotically). Thus, the robust price of anarchy is exactly $\frac{5+4 \alpha}{2+\alpha}$. We give a refinement of Theorem 4.1 to non-uniform altruism distributions, obtaining a bound in terms of the maximum and minimum altruism levels.

THEOREM 4.2. The robust price of anarchy of $\alpha$-altruistic linear congestion games is at most $\frac{5+2 \hat{\alpha}+2 \check{\alpha}}{2-\hat{\alpha}+2 \check{\alpha}}$.

As a first step in the proof of Theorem 4.2, we show that, without loss of generality, we can focus on simpler instances of linear congestion games. The proof is in Appendix B.

Lemma 4.3. Without loss of generality, all delay functions are of the form $d_{e}(x)=x$.

The next step in the proof of Theorem 4.2 is the following technical lemma:

Lemma 4.4. For every two nonnegative integers $x, y$ and $\hat{\alpha}, \check{\alpha} \in[0,1]$ with $\hat{\alpha} \geq \check{\alpha}$,

$$
((1+\hat{\alpha}) x+1) y+\check{\alpha}(1-x) x \leq \frac{5+2 \hat{\alpha}+2 \check{\alpha}}{3} y^{2}+\frac{1+\hat{\alpha}-2 \check{\alpha}}{3} x^{2} .
$$

To prove this lemma, we make use of the following result whose proof is also in Appendix B:

Lemma 4.5. For all $x, y \in \mathbb{N}_{0}, \eta \in[0,1]$ and $\beta \in[0,1]$, and all $\gamma \in\left[\frac{1}{3}(1+\eta-2 \beta \eta), 1+\eta\right]$,

$$
((1+\eta) x+1) y+\beta \eta(1-x) x \leq(2+\eta-\gamma) y^{2}+\gamma x^{2} .
$$

Now we can complete the proof of Lemma 4.4.

Proof of Lemma 4.4. Let $\beta=\frac{\check{\alpha}}{\hat{\alpha}} \in[0,1]$. Using Lemma 4.5, we obtain

$$
((1+\hat{\alpha}) x+1) y+\check{\alpha}(1-x) x=((1+\hat{\alpha}) x+1) y+\beta \hat{\alpha}(1-x) x \leq(2+\hat{\alpha}-\gamma) y^{2}+\gamma x^{2},
$$

for any $\gamma \in\left[\frac{1}{3}(1+\hat{\alpha}-2 \beta \hat{\alpha}), 1+\hat{\alpha}\right]$. By choosing $\gamma=\frac{1}{3}(1+\hat{\alpha}-2 \beta \hat{\alpha})$, we obtain that

$$
((1+\hat{\alpha}) x+1) y+\check{\alpha}(1-x) x \leq \frac{5+2 \hat{\alpha}+2 \beta \hat{\alpha}}{3} y^{2}+\frac{1+\hat{\alpha}-2 \beta \hat{\alpha}}{3} x^{2} .
$$

Substituting $\beta \hat{\alpha}=\check{\alpha}$ yields the claim.

We remark that the choice of $\gamma$ in the proof has been made in order to minimize the expression $\lambda /(1-\mu)$ (which is an increasing function in $\gamma$ ).

Proof of Theorem 4.2. We show that the $\alpha$-altruistic extension $G^{\alpha}$ of a linear congestion game is $\left(\frac{1}{3}(5+2 \hat{\alpha}+2 \check{\alpha}), \frac{1}{3}(1+\hat{\alpha}-2 \check{\alpha}), \boldsymbol{\alpha}\right)$-smooth.

Let $\boldsymbol{s}$ and $\boldsymbol{s}^{\prime}$ be two strategy profiles, and write $x_{e}=x_{e}(\boldsymbol{s}), x_{e}^{\prime}=x_{e}\left(\boldsymbol{s}^{\prime}\right)$. Using that $C_{-i}(\boldsymbol{s})=C(\boldsymbol{s})-C_{i}(\boldsymbol{s})$ by definition, the left-hand side of the smoothness condition (5) 
can be rewritten as

$$
\begin{aligned}
\sum_{i=1}^{n} & \left(\left(1-\alpha_{i}\right) C_{i}\left(s_{i}^{\prime}, \boldsymbol{s}_{-i}\right)+\alpha_{i}\left(C\left(s_{i}^{\prime}, \boldsymbol{s}_{-i}\right)-C(\boldsymbol{s})\right)+\alpha_{i} C_{i}(\boldsymbol{s})\right) \\
= & \sum_{i=1}^{n}\left(\left(1-\alpha_{i}\right)\left(\sum_{e \in s_{i}^{\prime} \backslash s_{i}}\left(x_{e}+1\right)+\sum_{e \in s_{i} \cap s_{i}^{\prime}} x_{e}\right)\right. \\
& \left.+\alpha_{i}\left(\sum_{e \in s_{i}^{\prime} \backslash s_{i}}\left(2 x_{e}+1\right)+\sum_{e \in s_{i} \backslash s_{i}^{\prime}}\left(1-2 x_{e}\right)\right)+\alpha_{i} C_{i}(\boldsymbol{s})\right) \\
= & \sum_{i=1}^{n}\left(\sum_{e \in s_{i}^{\prime} \backslash s_{i}}\left(\left(1+\alpha_{i}\right) x_{e}+1\right)+\left(1-\alpha_{i}\right) \sum_{e \in s_{i} \cap s_{i}^{\prime}} x_{e}+\alpha_{i} \sum_{e \in s_{i} \backslash s_{i}^{\prime}}\left(1-2 x_{e}\right)+\alpha_{i} \sum_{e \in s_{i}} x_{e}\right) \\
\leq & \sum_{i=1}^{n}\left(\sum_{e \in s_{i}^{\prime}}\left(\left(1+\alpha_{i}\right) x_{e}+1\right)+\alpha_{i} \sum_{e \in s_{i}}\left(1-2 x_{e}\right)+\alpha_{i} \sum_{e \in s_{i}} x_{e}\right) \\
= & \sum_{i=1}^{n}\left(\sum_{e \in s_{i}^{\prime}}\left(\left(1+\alpha_{i}\right) x_{e}+1\right)+\alpha_{i} \sum_{e \in s_{i}}\left(1-x_{e}\right)\right) \\
\leq & \sum_{e \in E}\left(\left((1+\hat{\alpha}) x_{e}+1\right) x_{e}^{\prime}+\check{\alpha}\left(1-x_{e}\right) x_{e}\right) .
\end{aligned}
$$

In this derivation, the first inequality follows from the fact that $\left(1-\alpha_{i}\right) x_{e} \leq\left(1+\alpha_{i}\right) x_{e}+$ $1+\alpha_{i}\left(1-2 x_{e}\right)$ for every $e \in s_{i} \cap s_{i}^{\prime}$. The second inequality holds because $1-x_{e} \leq 0$ for every $i \in N$ and $e \in s_{i}$, and by the definition of $\hat{\alpha}$ and $\check{\alpha}$. The bound on the robust price of anarchy now follows from Lemma 4.4 .

The following is a simple example that shows that the bound of $\frac{5+4 \alpha}{2+\alpha}$ on the robust price of anarchy for uniformly $\alpha$-altruistic linear congestion games is tight, even for pure Nash equilibria. It slightly improves the lower bound example of Caragiannis et al. [2010], because it is simpler and it shows tightness of the bound not only asymptotically.

Example 4.6. Consider a game with six resources $E=E_{1} \cup E_{2}, E_{1}=\left\{h_{0}, h_{1}, h_{2}\right\}, E_{2}=$ $\left\{g_{0}, g_{1}, g_{2}\right\}$ and three $\alpha$-altruistic players. The delay functions are $d_{e}(x)=(1+\alpha) x$ for $e \in E_{1}$, and $d_{e}(x)=x$ for $e \in E_{2}$. Each player $i$ has two pure strategies: $\left\{h_{i-1}, g_{i-1}\right\}$ and $\left\{h_{(i-2)(\bmod 3)}, h_{i(\bmod 3)}, g_{i(\bmod 3)}\right\}$. The strategy profile in which every player selects his first strategy is a social optimum of cost $(1+\alpha) \cdot 3+3=(2+\alpha) \cdot 3$.

Consider the strategy profile $s$ in which every player chooses his second strategy. We argue that $s$ is a Nash equilibrium. Each player's perceived individual cost is $c_{1}=(1-\alpha)(4(1+\alpha)+1)+\alpha(5+4 \alpha) \cdot 3$, whereas if a player unilaterally deviates to his first strategy, the new social cost would become $11 \alpha+16=(5+4 \alpha) \cdot 3+1-\alpha$. Thus, the player's new perceived individual cost is $c_{2}=(1-\alpha)(3(1+\alpha)+2)+\alpha((5+4 \alpha) \cdot 3+1-\alpha)$. Because $c_{1}=c_{2}, \boldsymbol{s}$ is a Nash equilibrium, of cost $4(1+\alpha) \cdot 3+3=(5+4 \alpha) \cdot 3$. We conclude that the pure price of anarchy is at least $\frac{5+4 \alpha}{2+\alpha}$ for $\alpha \in[0,1]$.

We turn to the pure price of stability of $\alpha$-altruistic congestion games. Notice that an upper bound on the pure price of stability extends to the mixed, correlated and coarse price of stability. 
Proposition 4.7. The pure price of stability of uniformly $\alpha$-altruistic linear congestion games is at most $\frac{2}{1+\alpha}$.

PROoF. The proof exploits a standard technique to bound the pure price of stability of exact potential games (see, e.g., Anshelevich et al. [2004] and Nisan et al. [2007]). Let $G^{\alpha}$ be a uniformly $\alpha$-altruistic extension of a linear congestion game. It is not hard to verify that $G^{\alpha}$ is an exact potential game with potential function $\Phi^{\alpha}(\boldsymbol{s})=(1-\alpha) \Phi(\boldsymbol{s})+\alpha C(\boldsymbol{s})$, where $\Phi(\boldsymbol{s})=\sum_{e \in E} \sum_{i=1}^{x_{e}(s)} i$ is Rosenthal's potential function [Rosenthal 1973]. Observe that for any strategy profile $s$,

$$
\begin{aligned}
\Phi^{\alpha}(\boldsymbol{s}) & =(1-\alpha) \sum_{e \in E} \sum_{i=1}^{x_{e}(\boldsymbol{s})} i+\alpha C(\boldsymbol{s}) \\
& =\frac{1-\alpha}{2} \sum_{e \in E}\left(f_{e}^{2}(\boldsymbol{s})+x_{e}(\boldsymbol{s})\right)+\alpha \sum_{e \in E} x_{e}^{2}(\boldsymbol{s})=\frac{1+\alpha}{2} C(\boldsymbol{s})+\frac{1-\alpha}{2} \sum_{e \in E} x_{e}(\boldsymbol{s}) .
\end{aligned}
$$

We therefore have $\frac{1+\alpha}{2} C(\boldsymbol{s}) \leq \Phi^{\alpha}(\boldsymbol{s}) \leq C(\boldsymbol{s})$. Now, let $\boldsymbol{s}^{*}$ be an optimal profile, minimizing $C\left(\boldsymbol{s}^{*}\right)$, and $\boldsymbol{s}$ a Nash equilibrium profile, minimizing $\Phi^{\alpha}(\boldsymbol{s})$. Then, $C(\boldsymbol{s}) \leq \frac{2}{1+\alpha} \Phi^{\alpha}(\boldsymbol{s}) \leq$ $\frac{2}{1+\alpha} \Phi^{\alpha}\left(\boldsymbol{s}^{*}\right) \leq \frac{2}{1+\alpha} C\left(\boldsymbol{s}^{*}\right)$, completing the proof.

\section{ATOMIC SYMMETRIC SINGLETON CONGESTION GAMES}

As defined in Section 2.1.1, symmetric singleton congestion games are the special case of congestion games in which $\Sigma_{i}=E$ for all players, that is, every strategy consists of a single resource, and each player has the same strategy space. In singleton linear congestion games, the focus here, delay functions are also assumed to be linear, that is, of the form $d_{e}(x)=a_{e} x+b_{e}$.

\subsection{Lower Bounds}

Caragiannis et al. [2010] prove the following theorem (stated using the transformation from Remark 3.2). It shows that the pure price of anarchy does not always increase with the altruism level; the relationship between $\alpha$ and the price of anarchy is thus rather subtle.

TheOREm 5.1 (CARAGIANNIS ET AL. [2010]). The pure price of anarchy of uniformly $\alpha$ altruistic singleton linear congestion games is $\frac{4}{3+\alpha}$.

We show that even the mixed price of anarchy (and thus also the robust price of anarchy) will be at least 2 regardless of the altruism levels of the players, by generalizing a result of Lücking et al. [2008, Theorem 5.4]. This implies that the benefits of higher altruism in singleton congestion games are only reaped in pure Nash equilibria, and the gap between the pure and mixed price of anarchy increases in $\alpha$. Also, it shows that singleton congestion games constitute a class of games for which the smoothness argument cannot deliver tight bounds.

Proposition 5.2. For every $\alpha \in[0,1]^{n}$, the mixed price of anarchy for $\alpha$-altruistic singleton linear congestion games is at least 2.

Proof. Let $m \geq 2$, and consider the instance with player set $\{1, \ldots, m\}$ and facility set $\{1, \ldots, m\}$, with $d_{e}(x)=x$ for each facility $e$. Denote by $\sigma$ the mixed strategy in which each player chooses each facility with probability $1 / \mathrm{m}$. When $\alpha_{i}=0$ for every player, $\sigma$ is a mixed Nash equilibrium, and $\mathbf{E}_{\boldsymbol{s} \sim \sigma}[C(\boldsymbol{s})]=2 m-1$, as proved in Lücking et al. [2008]. The optimum is clearly $m$, so the price of anarchy of this instance is $2-1 / \mathrm{m}$. 
All that is left to show is that $\sigma$ is also a Nash equilibrium under arbitrary altruism levels. By symmetry, it suffices to show that the expected perceived cost of player 1 increases if he deviates to the strategy where he chooses facility 1 with probability 1. In the following derivation, all expectations are taken over $s \sim \sigma$. By linearity of expectation,

$$
\mathbf{E}\left[C_{1}^{\alpha}\left(1, \boldsymbol{s}_{-1}\right)\right]=\left(1-\alpha_{1}\right) \mathbf{E}\left[C_{1}\left(1, \boldsymbol{s}_{-1}\right)\right]+\alpha_{1} \mathbf{E}\left[C\left(1, \boldsymbol{s}_{-1}\right)\right] .
$$

We already know that $\mathbf{E}\left[C_{1}\left(1, \boldsymbol{s}_{-1}\right)\right] \geq \mathbf{E}\left[C_{1}(\boldsymbol{s})\right]$, because $\sigma$ is a Nash equilibrium when the players are completely selfish, so it only remains to show that $\mathbf{E}\left[C\left(1, \boldsymbol{s}_{-1}\right)\right] \geq$ $\mathbf{E}[C(\boldsymbol{s})]=2 m-1$.

For an arbitrary pure strategy profile $\boldsymbol{s}^{\prime}$, let $X_{i, e}\left(\boldsymbol{s}^{\prime}\right)$ be the indicator function that maps to 1 if player $i$ chooses facility $e$ under $\boldsymbol{s}^{\prime}$, and 0 otherwise. Then, $C_{i}\left(\boldsymbol{s}^{\prime}\right)=\sum_{e} X_{i, e}\left(\boldsymbol{s}^{\prime}\right) d_{e}\left(\boldsymbol{s}^{\prime}\right)$ for $i=1, \ldots, m$, with $d_{e}\left(\boldsymbol{s}^{\prime}\right)=\sum_{i} X_{i, e}\left(\boldsymbol{s}^{\prime}\right)$ for $e=1, \ldots, m$. So $C_{i}\left(\boldsymbol{s}^{\prime}\right)=\sum_{e, j} X_{i, e}\left(\boldsymbol{s}^{\prime}\right) X_{j, e}\left(\boldsymbol{s}^{\prime}\right)$. Using this last identity, along with symmetry, independence, and linearity of expectation, we obtain the following derivation (letting $\boldsymbol{s}^{\prime}=\left(1, \boldsymbol{s}_{-1}\right)$ ):

$$
\begin{aligned}
\mathbf{E}\left[C\left(\boldsymbol{s}^{\prime}\right)\right]= & \sum_{i=1}^{m} \mathbf{E}\left[C_{i}\left(\boldsymbol{s}^{\prime}\right)\right]=\mathbf{E}\left[C_{1}\left(\boldsymbol{s}^{\prime}\right)\right]+(m-1) \mathbf{E}\left[C_{2}\left(\boldsymbol{s}^{\prime}\right)\right] \\
= & \mathbf{E}\left[d_{1}\left(\boldsymbol{s}^{\prime}\right)\right]+(m-1) \sum_{e, j=1}^{m} \mathbf{E}\left[X_{2, e}\left(\boldsymbol{s}^{\prime}\right) X_{j, e}\left(\boldsymbol{s}^{\prime}\right)\right] \\
= & \sum_{i=1}^{m} \mathbf{E}\left[X_{i, 1}\left(\boldsymbol{s}^{\prime}\right)\right]+(m-1)\left(\sum_{j=1}^{m} \mathbf{E}\left[X_{2,1}\left(\boldsymbol{s}^{\prime}\right) X_{j, 1}\left(\boldsymbol{s}^{\prime}\right)\right]\right) \\
& \quad+(m-1)\left(\sum_{j=1}^{m} \mathbf{E}\left[X_{2,2}\left(\boldsymbol{s}^{\prime}\right) X_{j, 2}\left(\boldsymbol{s}^{\prime}\right)\right]\right) \\
= & \left(1+(m-1) \frac{1}{m}\right)+(m-1)\left(\frac{1}{m}+\frac{1}{m}+(m-2) \frac{1}{m^{2}}+(m-1)\left(\frac{1}{m}+(m-2) \frac{1}{m^{2}}\right)\right) \\
= & 2 m-1 . \quad \square
\end{aligned}
$$

\subsection{Upper Bounds}

As a first step in the long-term goal to extend upper bounds to arbitrary non-uniform altruism distributions, we analyze the case when all altruism levels are in $\{0,1\}$, that is, each player is either completely altruistic or completely selfish. Then, the altruism distribution is entirely characterized by the fraction of altruistic players (which coincides with the average altruism level $\bar{\alpha}$ ). The next theorem shows that in this case, too, the pure price of anarchy improves with the overall altruism level.

THeOREM 5.3. Assume that an $\bar{\alpha}$ fraction of the players are completely altruistic, and the remaining $(1-\bar{\alpha})$ fraction are completely selfish. Then, the pure price of anarchy of the altruistic singleton linear congestion game is at most $\frac{4-2 \bar{\alpha}}{3-\bar{\alpha}}$.

Before proving Theorem 5.3, we note that the theorem implies that for linear singleton congestion games, entirely altruistic players will ensure that Nash equilibria are optimal. In fact, Lemma 5.5 implies that Nash equilibria reached by entirely altruistic players are optimal for singleton congestion games if the delay functions are semiconvex. We summarize this result in the following corollary. 
Corollary 5.4. The pure price of anarchy of 1-altruistic extensions of singleton congestion games with semiconvex delay functions is 1 .

We begin with a high-level outline of the proof of Theorem 5.3. Let $\boldsymbol{s}$ be a pure Nash equilibrium of $G^{\alpha}$, and $\boldsymbol{s}^{*}$ an optimal strategy profile. Again, we write $x_{e}=x_{e}(\boldsymbol{s})$ and $x_{e}^{*}=x_{e}\left(s^{*}\right)$. Based on the strategy profile $s$, we partition the facilities in $E$ into two sets $E_{0}$ and $E_{1}$ :

$$
\begin{aligned}
& E_{1}=\left\{e \in E: \exists i \in N \text { with } \alpha_{i}=1 \text { and } s_{i}=\{e\}\right\}, \\
& E_{0}=E \backslash E_{1} .
\end{aligned}
$$

That is, $E_{1}$ is the set of facilities having at least one altruistic player, while $E_{0}$ is the set of facilities that are used exclusively by selfish players or not used at all. Let $N_{1}$ and $N_{0}$ refer to the respective player sets that are using $E_{1}$ and $E_{0} . N_{1}$ may contain both altruistic and selfish players, while $N_{0}$ consists of selfish players only. Let $n_{1}=\left|N_{1}\right|=\sum_{e \in E_{1}} x_{e}$ and $n_{0}=\left|N_{0}\right|=n-n_{1}$ denote the number of players in $N_{1}$ and $N_{0}$, respectively.

The high-level approach of our proof is as follows: We split the total cost $C(\boldsymbol{s})$ of the pure Nash equilibrium into $C(\boldsymbol{s})=\gamma C(\boldsymbol{s})+(1-\gamma) C(\boldsymbol{s})$ for some $\gamma \in[0,1]$ such that $\gamma C(\boldsymbol{s})=\sum_{e \in E_{0}} x_{e} d_{e}\left(x_{e}\right)$ and $(1-\gamma) C(\boldsymbol{s})=\sum_{e \in E_{1}} x_{e} d_{e}\left(x_{e}\right)$. We bound these two contributions separately to show that

$$
\frac{3}{4} \gamma C(\boldsymbol{s})+(1-\gamma) C(\boldsymbol{s}) \leq C\left(\boldsymbol{s}^{*}\right)
$$

The pure price of anarchy is therefore at most $\left(\frac{3}{4} \gamma+(1-\gamma)\right)^{-1}=\frac{4}{4-\gamma}$. The bound then follows by deriving an upper bound on $\gamma$ in Lemma 5.7.

Our first lemma shows that in a sense, altruistic players "emulate" the social optimum. While we will only need the lemma for linear cost functions, it holds more generally for semi-convex functions, so we state and prove it in this generality.

LeMma 5.5. Assume that all cost functions $\left(d_{e}\right)_{e \in E}$ are semi-convex. Let $\boldsymbol{s}$ be a pure Nash equilibrium. Then, there is an optimal strategy profile $\boldsymbol{s}^{*}$ such that $x_{e}(\boldsymbol{s}) \leq x_{e}\left(\boldsymbol{s}^{*}\right)$ for every facility $e \in E_{1}$.

Proof. Assume that $x_{e}^{*}<x_{e}$ for some $e \in E_{1}$. Then, there is some facility $e^{\prime} \in E$ with $x_{e^{\prime}}^{*}>x_{e^{\prime}}$. Consider an altruistic player $i \in N_{1}$ with $s_{i}=\{e\}$. (Note that $i$ must exist by the definition of $E_{1}$.) Because $s$ is a pure Nash equilibrium, $i$ has no incentive to deviate from $e$ to $e^{\prime}$, that is, $C\left(\left\{e^{\prime}\right\}, \boldsymbol{s}_{-i}\right) \geq C(\boldsymbol{s})$, or, equivalently,

$$
\left(x_{e^{\prime}}+1\right) d_{e^{\prime}}\left(x_{e^{\prime}}+1\right)-x_{e^{\prime}} d_{e^{\prime}}\left(x_{e^{\prime}}\right) \geq x_{e} d_{e}\left(x_{e}\right)-\left(x_{e}-1\right) d_{e}\left(x_{e}-1\right) .
$$

Since $x_{e}^{*} \leq x_{e}-1$ and $x_{e^{\prime}} \leq x_{e^{\prime}}^{*}-1$, the semiconvexity of the delay functions implies that

$$
\begin{aligned}
\left(x_{e}^{*}+1\right) d_{e}\left(x_{e}^{*}+1\right)-x_{e}^{*} d_{e}\left(x_{e}^{*}\right) & \leq x_{e} d_{e}\left(x_{e}\right)-\left(x_{e}-1\right) d_{e}\left(x_{e}-1\right), \\
\left(x_{e^{\prime}}+1\right) d_{e^{\prime}}\left(x_{e^{\prime}}+1\right)-x_{e^{\prime}} d_{e^{\prime}}\left(x_{e^{\prime}}\right) & \leq x_{e^{\prime}}^{*} d_{e^{\prime}}\left(x_{e^{\prime}}^{*}\right)-\left(x_{e^{\prime}}^{*}-1\right) d_{e^{\prime}}\left(x_{e^{\prime}}^{*}-1\right) .
\end{aligned}
$$

By combining Inequalities (10), (11), and (12) and rearranging terms, we obtain that

$$
\left(x_{e}^{*}+1\right) d_{e}\left(x_{e}^{*}+1\right)+\left(x_{e^{\prime}}^{*}-1\right) d_{e^{\prime}}\left(x_{e^{\prime}}^{*}-1\right) \leq x_{e}^{*} d_{e}\left(x_{e}^{*}\right)+x_{e^{\prime}}^{*} d_{e^{\prime}}\left(x_{e^{\prime}}^{*}\right) .
$$

This inequality implies that by moving a player $j$ with $s_{j}^{*}=\left\{e^{\prime}\right\}$ from $e^{\prime}$ to $e$, we obtain a new strategy profile $\boldsymbol{s}^{\prime}=\left(\{e\}, \boldsymbol{s}^{*}{ }_{-j}\right)$ of $\operatorname{cost} C\left(\boldsymbol{s}^{\prime}\right) \leq C\left(\boldsymbol{s}^{*}\right)$. (Note that $j$ must exist because $x_{e^{\prime}}^{*}>x_{e^{\prime}} \geq 0$.) Moreover, the number of players using facility $e$ under the new strategy profile $\boldsymbol{s}^{\prime}$ increased by one. We can repeat the above argument (with $\boldsymbol{s}^{\prime}$ in place of $\boldsymbol{s}^{*}$ ) until we obtain an optimal strategy profile that satisfies the claim of the lemma. 
Henceforth, we assume, without loss of generality, that $\boldsymbol{s}^{*}$ is an optimal strategy profile satisfying the statement of Lemma 5.5. Next, we bound the cost that the Nash equilibrium incurs on the facilities that are not exclusively used by altruistic players.

Lemma 5.6. Define $y^{*}$ as $y_{e}^{*}=x_{e}^{*}-x_{e} \geq 0$ for every $e \in E_{1}$, and $y_{e}^{*}=x_{e}^{*}$ for every facility $e \in E_{0}$. Then, $\sum_{e \in E_{0}} x_{e} d_{e}\left(x_{e}\right) \leq \frac{4}{3} \sum_{e \in E} y_{e}^{*} d_{e}\left(x_{e}^{*}\right)$.

Proof. Consider the game $\bar{G}$ induced by $G^{\alpha}$ if all $n_{1}$ players in $N_{1}$ are fixed on the facilities in $E_{1}$ according to $\boldsymbol{s}$. Note that all remaining $n_{0}=n-n_{1}$ players in $N_{0}$ are selfish. That is, $\bar{G}$ is a symmetric singleton congestion game with player set $N_{0}$, facility set $E$ and delay functions $\left(\bar{d}_{e}\right)_{e \in E}$, where $\bar{d}_{e}(z)=d_{e}\left(x_{e}+z\right)$ if $e \in E_{1}$ and $\bar{d}_{e}(z)=d_{e}(z)$ for $e \in E_{0}$. Let $\overline{\boldsymbol{s}}$ be the restriction of $\boldsymbol{s}$ to the players in $N_{0}$, and define $\bar{x}$ to be the induced flows: that is, $\bar{x}_{e}=0$ for $e \in E_{1}$ and $\bar{x}_{e}=x_{e}$ for $e \in E_{0}$.

$\overline{\boldsymbol{s}}$ is a pure Nash equilibrium of the game $\bar{G}$, because each of the selfish players in $N_{0}$ faces exactly the same situation in $\bar{G}$ under $\overline{\boldsymbol{s}}$ as in $G^{\alpha}$ under $\boldsymbol{s}$. Let $\overline{\boldsymbol{s}}^{*}$ be a socially optimum profile for $\bar{G}$; for each facility $e$, let $\bar{x}_{e}^{*}$ be the total number of players on $e$ under $\overline{\boldsymbol{s}}^{*}$. Then,

$$
\sum_{e \in E_{0}} x_{e} d_{e}\left(x_{e}\right)=\sum_{e \in E} \bar{x}_{e} \bar{d}_{e}\left(\bar{x}_{e}\right) \leq \frac{4}{3} \sum_{e \in E} \bar{x}_{e}^{*} \bar{d}_{e}\left(\bar{x}_{e}^{*}\right) \leq \frac{4}{3} \sum_{e \in E} y_{e}^{*} \bar{d}_{e}\left(y_{e}^{*}\right)=\frac{4}{3} \sum_{e \in E} y_{e}^{*} d_{e}\left(x_{e}^{*}\right) .
$$

The first inequality follows from Theorem 5.1, and the second inequality follows from the optimality of $\overline{\boldsymbol{s}}^{*}$.

LEMMA 5.7. $\gamma$ is bounded by $\gamma \leq \frac{2 n_{0}}{n+n_{0}} \leq \frac{2(1-\bar{\alpha})}{2-\bar{\alpha}}$.

Proof. The claim follows directly from Theorem 5.1 if $N_{1}=\emptyset$, and it holds trivially whenever $N_{0}=\emptyset$ by definition of $\gamma$. So assume that $N_{0} \neq \emptyset, N_{1} \neq \emptyset$, and let $j \in N_{1}$ be arbitrary with $s_{j}=\left\{e^{\prime}\right\}, e^{\prime} \in E_{1}$. Let $\bar{C}(\boldsymbol{s})=\sum_{i \in N_{0}} C_{i}(\boldsymbol{s}) / n_{0}$ be the average cost experienced by the (selfish) players in $N_{0}$. We first show $C_{j}(\boldsymbol{s}) \geq \frac{1}{2} \bar{C}(\boldsymbol{s})$. Let $i \in N_{0}$ be arbitrary, $s_{i}=\{e\}, e \in E_{0}$. Because $s$ is a Nash equilibrium, and $i$ does not want to deviate to facility $e^{\prime}$,

$$
C_{i}(\boldsymbol{s})=a_{e} x_{e}+b_{e} \leq a_{e^{\prime}}\left(x_{e^{\prime}}+1\right)+b_{e^{\prime}} \leq 2\left(a_{e^{\prime}} x_{e^{\prime}}+b_{e^{\prime}}\right)=2 C_{j}(\boldsymbol{s}) .
$$

By summing over all $n_{0}$ selfish players in $N_{0}$, we obtain that $C_{j}(s) \geq \frac{1}{2} \bar{C}(s)$, and thus $\sum_{j \in N_{1}} C_{j}(\boldsymbol{s}) \geq \frac{1}{2} n_{1} \bar{C}(\boldsymbol{s})$. By definition of $\gamma$,

$$
\gamma=\frac{\sum_{i \in N_{0}} C_{i}(\boldsymbol{s})}{\sum_{i \in N_{0}} C_{i}(\boldsymbol{s})+\sum_{j \in N_{1}} C_{j}(\boldsymbol{s})} \leq \frac{n_{0} \bar{C}(\boldsymbol{s})}{n_{0} \bar{C}(\boldsymbol{s})+\frac{1}{2} n_{1} \bar{C}(\boldsymbol{s})}=\frac{2 n_{0}}{n+n_{0}} \leq \frac{2(1-\bar{\alpha})}{2-\bar{\alpha}},
$$

where the last inequality follows because $n_{0} \leq(1-\bar{\alpha}) n$.

Proof of Theorem 5.3. Using these lemmas, and the definition of $y_{e}^{*}$ from Lemma 5.6, we can prove Inequality (9):

$$
\begin{aligned}
\frac{3}{4} \gamma C(\boldsymbol{s})+(1-\gamma) C(\boldsymbol{s}) & =\frac{3}{4} \sum_{e \in E_{0}} x_{e} d_{e}\left(x_{e}\right)+\sum_{e \in E_{1}} x_{e} d_{e}\left(x_{e}\right) \leq \sum_{e \in E} y_{e}^{*} d_{e}\left(x_{e}^{*}\right)+\sum_{e \in E_{1}} x_{e} d_{e}\left(x_{e}\right) \\
& =\sum_{e \in E} x_{e}^{*} d_{e}\left(x_{e}^{*}\right)+\sum_{e \in E_{1}}\left(x_{e} d_{e}\left(x_{e}\right)-x_{e} d_{e}\left(x_{e}^{*}\right)\right) \leq \sum_{e \in E} x_{e}^{*} d_{e}\left(x_{e}^{*}\right)=C\left(\boldsymbol{s}^{*}\right) .
\end{aligned}
$$

The first inequality follows from Lemma 5.6, and the last inequality follows from Lemma 5.5 and because cost functions are monotone nondecreasing. We use Lemma 5.7 
to conclude that the pure price of anarchy is at most

$$
\left(\frac{3}{4} \gamma+(1-\gamma)\right)^{-1}=\frac{4}{4-\gamma} \leq \frac{4-2 \bar{\alpha}}{3-\bar{\alpha}}
$$

\section{NONATOMIC CONGESTION GAMES}

In this section, we turn our attention to studying nonatomic congestion games, as defined in Section 2.1.2. We first prove that such games always have Nash equilibria for arbitrary altruism distributions and delay functions.

Theorem 6.1. Each instance $\left(E, \boldsymbol{r}, \boldsymbol{d},\left(\psi_{i}\right)\right)$ of a nonatomic congestion game with (nonuniform) altruism has a Nash equilibrium.

Proof. Theorem 1 of Mas-Colell [1984] proves that each game of infinitely many players has a Nash equilibrium. A game is characterized by a distribution (Borel measure) over utility functions which are continuous in the action of the player and the distribution of actions by the remaining players. It is easy to see that each player in the congestion game has a utility function $-d_{S}^{(\alpha)}(f)$ continuous in the choice of strategy $S \in \mathcal{S}$ (trivially, since each strategy space $\mathcal{S}$ is finite) and in the distribution of other players' strategies $f$ (by continuity of each $d_{e}$ ). The utility for sets $S \notin \mathcal{S}$ is $-\infty$ (or a suitably negative constant). The distribution of altruism values $\alpha$ implies a corresponding distribution over utility functions. Thus, the theorem of Mas-Collel [1984] implies the existence of Nash equilibria for nonatomic congestion games.

The proof of Mas-Collel is inherently nonconstructive; accordingly, Theorem 6.1 does not imply any algorithm for finding such equilibria. Indeed, even if the altruism distribution has support of size 2, we are not aware of an algorithm for finding a Nash equilibrium.

Another difficulty arising for general altruism distributions is that the price of anarchy can easily become unbounded. Indeed, even if the altruism distribution has support $\{0,1\}$ with an arbitrarily large average altruism level $\bar{\alpha}<1$, a result on Stackelberg routing due to Bonifaci et al. [2007] implies that the price of anarchy can become unbounded. This result applies even in the case when facilities are edges of a graph, and all players are of the same type, with feasible sets consisting of all $s$ - $t$ paths for a fixed pair $(s, t)$ of nodes.

In order to address this prohibitive negative result, we focus on two special cases: first, we consider the case of uniform altruism; subsequently, we focus on symmetric singleton congestion games with arbitrary altruism distributions (see Section 7).

\subsection{Uniform Altruism}

In this section, we focus on the case of uniformly altruistic players. Furthermore, we assume that all latency functions $d_{e}$ are semiconvex. Then, there is an explicit characterization of pure Nash equilibria via a convex program.

Proposition 6.2. Let $(E, \boldsymbol{r}, \boldsymbol{d}, \alpha)$ be an instance with uniform altruism $\alpha \geq 0$ and semiconvex latency functions $d_{e}$. Then, the pure Nash equilibria are the optima of the convex program

$$
\begin{aligned}
& \text { Minimize } \sum_{e} \int_{0}^{f_{e}} d_{e}^{(\alpha)}(t) d t \\
& \text { subject to } f \text { is a feasible solution for }(E, \boldsymbol{r}) \text {. }
\end{aligned}
$$

The proof of this proposition is virtually identical to that of Proposition 2.6.1 from Roughgarden [2005]. The proof there only uses the fact that each agent is minimizing a sum of monotone increasing functions $\sum_{e} g_{e}\left(f_{e}\right)$, in order to conclude that the Nash 
equilibrium minimizes the (convex) objective $\sum_{e} \int_{0}^{f_{e}} g_{e}(t) d t$. Thus, it applies equally to $g_{e}(t):=d_{e}^{(\alpha)}(t)$.

When there is an efficient oracle for feasible solutions for $(E, \boldsymbol{r})$, the convex program can also be solved in polynomial time. In particular, this is the case when $E$ is the set of edges in a graph, and the feasible sets of player type $i$ are paths from $s_{i}$ to $t_{i}$, for some source node $s_{i}$ and sink $t_{i}$. In that case, feasible solutions are exactly multi-commodity flows of rates $r_{i}$.

Nash equilibria can be characterized by the following variational inequality, which we will use below. The proof is in Appendix C.

Proposition 6.3. Let $(E, \boldsymbol{r}, \boldsymbol{d}, \alpha)$ be an instance with uniform altruism $\alpha \geq 0$. Then, $f$ is a Nash equilibrium for $\alpha$-altruistic players if and only if it minimizes $\sum_{S \subseteq E} d_{S}^{(\alpha)}(f) \tilde{f}_{S}$ over all solutions $\tilde{f}$ feasible for $(E, \boldsymbol{r})$.

As an easy warm-up, we give a simple proposition bounding the pure price of anarchy for arbitrary congestion games with semiconvex delay functions.

Proposition 6.4. If all delay functions $d_{e}$ are nondecreasing and semiconvex, then for all congestion games, and any altruism level $\alpha \in(0,1]$, the pure price of anarchy is at most $1 / \alpha$.

Proof. Let $\hat{f}$ be a pure Nash equilibrium flow, minimizing the potential function $\Phi(f)=\sum_{e} \int_{0}^{f_{e}} d_{e}^{(\alpha)}(t) d t$, the objective function of the convex program in Proposition 6.2. Also, let $f^{*}$ be the optimum flow, minimizing the total cost $C(f)=\sum_{e} \int_{0}^{f_{e}}\left(t d_{e}(t)\right)^{\prime} d t$. Simply from the definition of $d_{e}^{(\alpha)}(t)$, it follows that for any flow $f$, we have $\Phi(f) \leq$ $C(f) \leq \frac{1}{\alpha} \Phi(f)$. Applying the first inequality to $f^{*}$ and the second to $\hat{f}$, and using the optimality of $\hat{f}$ for $\Phi$, we obtain

$$
C(\hat{f}) \leq \frac{1}{\alpha} \Phi(\hat{f}) \leq \frac{1}{\alpha} \Phi\left(f^{*}\right) \leq \frac{1}{\alpha} C\left(f^{*}\right) .
$$

More generally, we derive a result bounding the robust price of anarchy when all delay functions $d_{e}$ are drawn from a given class of delay functions. As a special case of this general result, we will derive the same bound of $1 / \alpha$ as a robust price of anarchy when the delay functions are arbitrary increasing semiconvex functions. Our characterization will be in terms of the anarchy value $\sigma^{(\alpha)}(\mathcal{C})$ of a set $\mathcal{C}$ of functions for $\alpha$-altruistic players, which is defined as a generalization of the anarchy value of functions in Roughgarden [2005].

\section{Definition 6.5.}

(1) For any delay function $d$, the anarchy value $\sigma^{(\alpha)}(d)$ of $d$ for $\alpha$-altruistic players is defined as

$$
\sigma^{(\alpha)}(d)=\sup _{r, x \geq 0} \frac{r \cdot d(r)}{x \cdot d(x)+(r-x) \cdot d^{(\alpha)}(r)},
$$

where $0 / 0$ is defined to be 1 .

(2) For any class $\mathcal{C}$ of delay functions, the anarchy value for $\alpha$-altruistic players $\sigma^{(\alpha)}(\mathcal{C})$ is

$$
\sup _{d \in \mathcal{C}, d \neq 0} \sigma^{(\alpha)}(d) .
$$

The motivation for this definition of $\sigma^{(\alpha)}(d)$ is that it captures the price of anarchy for uniformly $\alpha$-altruistic symmetric players with two facilities, where one facility has 
delay function $d$ and the other has a worst-case constant. Indeed, we will prove this to be the case in Lemma 6.10. Notice that Lemma 6.10 immediately implies that $\sigma^{(\alpha)}(\mathcal{C})$ is a lower bound on the pure price of anarchy in the worst case when all facility delay functions are chosen from $\mathcal{C}$. Our main theorem in this section shows that it is also an upper bound on the coarse price of anarchy for all networks and arbitrary commodities.

THEOREM 6.6. Let $\mathcal{C}$ be a set of delay functions, and $G=(E, \boldsymbol{r}, \boldsymbol{d}, \alpha)$ an instance with delay functions $d_{e} \in \mathcal{C}$ and uniform altruism $\alpha$. Then, the coarse price of anarchy is at most $\sigma^{(\alpha)}(\mathcal{C})$.

Proof. Let $f, f^{\prime}$ be any two feasible flows. By rearranging Definition 6.5, we obtain the bound

$$
x \cdot d_{e}(x) \geq \frac{r \cdot d_{e}(r)}{\sigma^{(\alpha)}(\mathcal{C})}+(x-r) \cdot d_{e}^{(\alpha)}(r)
$$

for any $x, r \geq 0$. Applying this bound to each facility $e$, with $x=f_{e}^{\prime}$ and $r=f_{e}$, we get that

$$
\begin{aligned}
C\left(f^{\prime}\right) & =\sum_{e \in E} f_{e}^{\prime} d_{e}\left(f_{e}^{\prime}\right) \\
& \geq \frac{1}{\sigma^{(\alpha)}(\mathcal{C})} \cdot \sum_{e \in E} f_{e} d_{e}\left(f_{e}\right)+\sum_{e \in E}\left(f_{e}^{\prime}-f_{e}\right) \cdot d_{e}^{(\alpha)}\left(f_{e}\right) \\
& =\frac{C(f)}{\sigma^{(\alpha)}(\mathcal{C})}+\sum_{e \in E}\left(f_{e}^{\prime}-f_{e}\right) \cdot\left(d_{e}\left(f_{e}\right)+\alpha f_{e} d_{e}^{\prime}\left(f_{e}\right)\right) \\
& =C(f) \cdot\left(\frac{1}{\sigma^{(\alpha)}(\mathcal{C})}-1\right)+\sum_{e \in E}\left(f_{e}^{\prime} d_{e}\left(f_{e}\right)+\left(f_{e}^{\prime}-f_{e}\right) \cdot \alpha f_{e} d_{e}^{\prime}\left(f_{e}\right)\right) .
\end{aligned}
$$

Rearranging gives us that

$$
\sum_{e \in E}\left(f_{e}^{\prime} d_{e}\left(f_{e}\right)+\left(f_{e}^{\prime}-f_{e}\right) \cdot \alpha f_{e} d_{e}^{\prime}\left(f_{e}\right)\right) \leq C\left(f^{\prime}\right)+\left(1-\frac{1}{\sigma^{(\alpha)}(\mathcal{C})}\right) \cdot C(f) .
$$

Hence, the game is $\left(1,1-\frac{1}{\sigma^{(\alpha)}(\mathcal{C})}, \alpha\right)$-smooth, and Proposition 3.6 implies that the coarse price of anarchy is at most $\sigma^{(\alpha)}(\mathcal{C})$.

As a corollary of Theorem 6.6 whose proof is in Appendix C, we obtain a tight bound in the case where the delay functions are polynomials of degree at most $p$ with nonnegative coefficients. We denote this class by $\mathcal{C}_{p}$.

THEOREM 6.7. If $(E, \boldsymbol{r}, \boldsymbol{d}, \alpha)$ has delay functions in $\mathcal{C}_{p}$, then the coarse price of anarchy is at most

$$
\left(\left(\frac{1+\alpha p}{1+p}\right)^{1 / p}\left(\frac{1+\alpha p}{1+p}-1-\alpha p\right)+1+\alpha p\right)^{-1}
$$

It is not difficult to verify that the previous bound converges to $\frac{1}{\alpha}$ as $p \rightarrow \infty$; the worst-case behavior is in fact attained with polynomials of high degree. However, for $p=1$, Theorem 6.7 also allows us to obtain a tighter bound in the special case that all delay functions are linear.

COROLlary 6.8. If $(E, \boldsymbol{r}, \boldsymbol{d}, \alpha)$ has linear delay functions, then the coarse price of anarchy is at most $\frac{4}{3+2 \alpha-\alpha^{2}}$. 
Remark 6.9. Notice that for any $\alpha>0$, this bound improves on the bound by Roughgarden and Tardos [2000] of $4 / 3$ when all players are completely selfish. As the bound is also shown to be tight, it characterizes exactly the gain by altruism with linear delay functions. Also notice that the bound for nonatomic games is significantly better than the bound of $\frac{5+4 \alpha}{2+\alpha}$ from Theorem 4.1 for arbitrary atomic linear congestion games, and slightly better than the bound of $4 /(3+\alpha)$ from Theorem 5.1. This improved bound results from the fact that players are nonatomic, avoiding the cost of slight "rounding issues."

Finally, we show that the bounds derived in Theorem 6.6 are indeed tight, even for the pure price of anarchy in symmetric singleton congestion games with two facilities:

LEMMA 6.10. Consider a symmetric singleton congestion game with two facilities and flow rate $r=1$. Let the delay functions be $d_{1}(x)=d(x)$ for the first facility, and the constant delay function $d_{2}(x)=d^{(\alpha)}(1)=d(1)+\alpha d^{\prime}(1)$ for the second facility. The pure price of anarchy of this instance is $\sigma^{(\alpha)}(d)$.

Proof. It is easy to observe from the definition of $d_{2}$ that all $\alpha$-altruistic players will end up using facility 1 , so that the total cost of the Nash equilibrium is $d(1)$, while the socially optimum solution has total cost

$$
\inf _{x \leq 1}\left(x \cdot d(x)+(1-x) \cdot d(1)+\alpha(1-x) d^{\prime}(1)\right) .
$$

Hence, the pure price of anarchy is exactly $\sigma(d)$.

By applying this characterization together with Theorem 6.7 and letting the degree of the polynomial go to $\infty$, we obtain instances $(E, \boldsymbol{r}, \boldsymbol{d}, \alpha)$ whose price of anarchy approaches $1 / \alpha$ arbitrarily closely. Similarly, by choosing $p=1$, we obtain that the bound in Corollary 6.8 is tight.

\section{NONATOMIC SYMMETRIC SINGLETON CONGESTION GAMES}

We next extend our study to the case of arbitrary distributions $\psi$ of altruism. In light of the negative result of Bonifaci et al. [2007], who proved an unbounded price of anarchy even for the case of symmetric congestion games, we focus on the case of symmetric singleton congestion games. Recall that such games naturally model the assignment of infinitesimally small jobs to machines with load-dependent delays. Our main theorem in this section gives a (tight) upper bound on the pure price of anarchy in nonatomic symmetric singleton congestion games with arbitrary sets of (convex) delay functions closed under addition of constants.

THEOREM 7.1. Let $\mathcal{C}$ be a set of convex and nondecreasing functions closed under addition of constants; that is, if $d \in \mathcal{C}$, then $\hat{d}(x)=d(x)+c$ is in $\mathcal{C}$ for every constant $c$. Then, for every nonatomic symmetric singleton congestion game (with arbitrary altruism distribution $\psi$ ), the pure price of anarchy is at most

$$
\left(\int_{0}^{1} \psi(t) \frac{1}{\sigma^{(t)}(\mathcal{C})} d t\right)^{-1}
$$

Before proving Theorem 7.1, we observe and prove several interesting corollaries. First, we obtain a much simplified bound when $\mathcal{C}$ is the set of all semiconvex nondecreasing functions.

COROLlaRY 7.2. If all delay functions $d_{e}$ are semiconvex and nondecreasing, then for any nonatomic symmetric singleton congestion game with arbitrary altruism density 
distribution $\psi$, the pure price of anarchy is at most $1 / \bar{\alpha}$, where $\bar{\alpha}$ is the average altruism under $\psi$.

PRoOF. If $\mathcal{C}$ is specifically the set of all increasing semiconvex functions, Proposition 6.4 implies that $\frac{1}{\sigma^{(t)}(\mathcal{C})} \geq t$. Substituting this bound into the integral in Theorem 7.1 gives us that the pure price of anarchy is at most $\left(\int_{0}^{1} \psi(t) t d t\right)^{-1}=1 / \bar{\alpha}$.

In turn, an immediate corollary of Corollary 7.2 can be obtained by choosing the distribution $\psi$ to have a $\lambda$ fraction of completely altruistic players, and a $1-\lambda$ fraction of completely selfish ones. As discussed in Section 1.3, such a scenario corresponds to a Stackelberg scheduling game with a centrally controlled fraction $\lambda$ of jobs. Since $\bar{\alpha}=\lambda$ for this distribution, Corollary 7.2 immediately implies:

COROLlary 7.3. In symmetric singleton congestion games, the pure price of anarchy under Stackelberg scheduling with a $\lambda$-fraction of jobs being controlled by a central authority is at most $1 / \lambda$.

This result was already proved constructively (and giving efficient algorithms) by Roughgarden [2004]; nevertheless, it is interesting that it follows directly from our general result. More generally, by using the same distribution with support $\{0,1\}$ in Theorem 7.1, we obtain the following corollary:

COROLlary 7.4. In symmetric singleton congestion games, the pure price of anarchy under Stackelberg scheduling with a $\lambda$-fraction of jobs controlled by a central authority is at most $\left(\frac{1-\lambda}{\sigma(\mathcal{C})}+\lambda\right)^{-1}$.

Corollary 7.4 improves (albeit in a nonconstructive way) a result of Swamy [2007] for Stackelberg scheduling: we bound the PoA under Stackelberg scheduling by the weighted harmonic mean of the PoA for selfish and altruistic players, whereas Swamy's bounds give the arithmetic mean. It is known that the harmonic mean is always bounded above by the arithmetic mean.

We can also show that the case of Stackelberg scheduling is in fact the worst case for the bound of Theorem 7.1, in the sense that the right-hand side is maximized. While the bound of Theorem 7.1 will in general not be tight, this nevertheless gives rise to the philosophical interpretation that, conditioned on a given average altruism level $\bar{\alpha}$, the scenario in which completely altruistic players or a central authority compensate for completely selfish players is the worst case, while uniform altruism throughout the population is the best case. The proof is in Appendix D.

Proposition 7.5. Conditioned on the mean of $\psi$ being any given $\bar{\alpha}$, the quantity $\left(\int_{0}^{1} \psi(t) \frac{1}{\sigma^{(t)}(\mathcal{C})} d t\right)^{-1}$ is maximized when $\psi$ has a point mass of $\bar{\alpha}$ on 1 and $1-\bar{\alpha}$ on 0 . It is minimized when $\psi$ has a point mass of 1 on $\bar{\alpha}$.

\subsection{Proof of Theorem 7.1}

The remainder of this section is devoted to the proof of Theorem 7.1. The proof's structure is similar to that of Theorem 5.3, although the proof is technically more involved, since we allow arbitrary altruism distributions, while Theorem 5.3 only considered altruism levels in $\{0,1\}$.

Let $f$ be a pure Nash equilibrium flow. We first show that without loss of generality, we can assume that each facility $e$ contains only one type of players (i.e., if players have different altruism values $\alpha, \alpha^{\prime}$, then they do not share a facility) and that the support of $\psi$ is finite. To see this, assume that $f$ has players of altruism values $\alpha<\alpha^{\prime}$ sharing a facility $e$. Now replace all players on $e$ with altruism $\alpha$ by players with altruism $\alpha^{\prime}$. $f$ must still be a flow at Nash equilibrium for the new instance (because $\alpha^{\prime}$-altruistic 
players are on facility $e$ in Nash equilibrium). By repeating this process, we eventually obtain an instance with altruism density $\psi^{\prime}$ which stochastically dominates $\psi$ and has finite support. For this new $\psi^{\prime}$, the bound on the price of anarchy for $f$ provided by the right-hand side of Theorem 7.1 can only be smaller, giving us an even better bound than required. Thus, we can from now on focus on the case described here.

Let $0 \leq \alpha_{1}<\alpha_{2}<\cdots<\alpha_{k} \leq 1$ be the (finite) support of $\psi$, where the rate of $\alpha_{i}$ altruistic players is $r_{i}$ (so $\sum_{i=1}^{k} r_{i}=r$ ). We need to show that for all flows $g$ of rate $r$ (in particular, the optimum flow), we have

$$
C(g) \geq C(f) \sum_{i=1}^{k} \frac{r_{i}}{r} \frac{1}{\sigma^{\left(\alpha_{i}\right)}(\mathcal{C})},
$$

which we will do by induction on $k$. The base case $k=0$ is of course trivial.

For the inductive step, let $f$ be a Nash equilibrium, and $g$ any flow of rate $r$. For each $i$, let $E_{i}$ be the set of facilities with positive flow of $\alpha_{i}$-altruistic players under $f$. Notice that, by our assumption, the sets $E_{i}$ are pairwise disjoint. For any set $E^{\prime}$ of facilities, let $f\left(E^{\prime}\right)=\sum_{e \in E^{\prime}} f_{e}$ (similarly, $\left.g\left(E^{\prime}\right)\right)$ denote the total flow on $E^{\prime}$. Let $E^{\prime}:=E \backslash E_{1}$ denote the set of all facilities not used by $\alpha_{1}$-altruistic players.

Intuitively, because the more altruistic players prefer the facilities in $E^{\prime}$ over $E_{1}$, we would expect a "good" flow $g$ to do the same. Indeed, we first show that the delay under $f$ on all facilities in $E_{1}$ is no larger than in $E^{\prime}$, while the marginal cost, that is, $(x \cdot d(x))^{\prime}$, is no larger in $E^{\prime}$ than in $E_{1}$. Let $e \in E_{1}, e^{\prime} \in E_{j}, j>1$ be arbitrary facilities with positive flow $f$. Thus, all players using $e$ have altruism $\alpha_{1}$, while all players using $e^{\prime}$ have altruism $\alpha_{j}>\alpha_{1}$. Because $f$ is at Nash equilibrium,

$$
\begin{aligned}
& d_{e}\left(f_{e}\right)+\alpha_{1} f_{e} d_{e}^{\prime}\left(f_{e}\right) \leq d_{e^{\prime}}\left(f_{e^{\prime}}\right)+\alpha_{1} f_{e^{\prime}} d_{e^{\prime}}^{\prime}\left(f_{e^{\prime}}\right), \\
& d_{e}\left(f_{e}\right)+\alpha_{j} f_{e} d_{e}^{\prime}\left(f_{e}\right) \geq d_{e^{\prime}}\left(f_{e^{\prime}}\right)+\alpha_{j} f_{e^{\prime}} d_{e^{\prime}}^{\prime}\left(f_{e^{\prime}}\right) .
\end{aligned}
$$

Combining appropriately scaled versions of (14) and (15) gives us that

$$
\begin{aligned}
d_{e}\left(f_{e}\right) & \leq d_{e^{\prime}}\left(f_{e^{\prime}}\right), \\
(1-\xi) d_{e}\left(f_{e}\right)+\left(\alpha_{j}-\xi \alpha_{1}\right) f_{e} d_{e}^{\prime}\left(f_{e}\right) & \geq(1-\xi) d_{e^{\prime}}\left(f_{e^{\prime}}\right)+\left(\alpha_{j}-\xi \alpha_{1}\right) f_{e^{\prime}} d_{e^{\prime}}^{\prime}\left(f_{e^{\prime}}\right),
\end{aligned}
$$

where $\xi \in[0,1]$ is a scalar, which we can set later.

Our high-level strategy will be to bound the Nash equilibrium flow on $E^{\prime}$ against a restriction $g^{\prime}$ of $g$ of rate $r-r_{1}$ on $E^{\prime}$ by induction, and use a comparison argument for the flow on $E_{1}$. We will construct a flow $h$ of rate $r_{1}$ whose cost is cheaper than a component of $g$ of the same rate, and which is optimal for modified "residual" facility costs. We can thus compare it against the flow $f$ on $E_{1}$ using Theorem 6.6.

Define $f^{\prime}$ to be the restriction of $f$ to the set $E^{\prime}$, that is, $f_{e}^{\prime}=f_{e}$ for $e \in E^{\prime}$, and $f_{e}^{\prime}=0$ for $e \in E_{1}$. Thus, $f^{\prime}$ is a flow of rate $r^{\prime}:=r-r_{1}$. Define the modified delay function

$$
\tilde{d}_{e}(x):=d_{e}\left(f_{e}^{\prime}+x\right)+\alpha_{1} f_{e}^{\prime} d_{e}^{\prime}\left(f_{e}^{\prime}\right)
$$

for all facilities $e$. Thus, $\tilde{d}_{e}(x)$ is the delay incurred by flow on $e$ if $f_{e}^{\prime}$ is unalterable, but not considered part of the actual flow, plus a suitable constant term to "mimic" the altruistic component. This definition of $\tilde{d}_{e}(x)$ implies that the perceived cost of facility $e$ to $\alpha_{1}$-altruistic players is

$$
\tilde{d}_{e}^{\left(\alpha_{1}\right)}(x)=d_{e}\left(f_{e}^{\prime}+x\right)+\alpha_{1} x d_{e}^{\prime}\left(f_{e}^{\prime}+x\right)+\alpha_{1} f_{e}^{\prime} d_{e}^{\prime}\left(f_{e}^{\prime}\right) .
$$

Thus, for $e \in E^{\prime}$, we have that $\tilde{d}_{e}^{\left(\alpha_{1}\right)}(x) \geq d_{e}^{\left(\alpha_{1}\right)}\left(f_{e}^{\prime}\right)$ for all $x \geq 0$, while for $e \in E_{1}$, because $f_{e}^{\prime}=0, \tilde{d}_{e}^{\left(\alpha_{1}\right)}(x)=d_{e}^{\left(\alpha_{1}\right)}\left(x+f_{e}^{\prime}\right)$. In particular, this implies that the $\alpha_{1}$-altruistic players are at Nash equilibrium with respect to the modified delay functions $\tilde{d}_{e}(x)$. Hence, by 
Theorem 6.6, and because $\tilde{d}_{e}(x)=d_{e}(x)$ for all $e \in E_{1}$, we get

$$
C\left(f-f^{\prime}\right)=\tilde{C}\left(f-f^{\prime}\right) \leq \sigma^{\left(\alpha_{1}\right)}(\mathcal{C}) \cdot \tilde{C}(\tilde{f}),
$$

where $\tilde{f}$ is an optimum flow of rate $r_{1}$ with respect to the modified delay functions $\tilde{d}_{e}$.

In order to compare $f^{\prime}$ against the part of $g$ on the set $E^{\prime}$, it will be useful to assume that $g\left(E^{\prime}\right) \geq f\left(E^{\prime}\right)$. We will show next that we can make this assumption without loss of generality. For assume that it did not hold. Then, let $e \in E_{1}, e^{\prime} \in E^{\prime}$ be facilities with $g_{e}>f_{e}>0$ and $g_{e^{\prime}}<f_{e^{\prime}}$. (The existence of $e, e^{\prime}$ follows from the assumption $\left.g\left(E^{\prime}\right)<f\left(E^{\prime}\right)\right)$. By the bound on the derivatives in Inequality (17), and using the semiconvexity of the facility cost functions, we show that

$$
\left(g_{e} d_{e}\left(g_{e}\right)\right)^{\prime} \geq\left(f_{e} d_{e}\left(f_{e}\right)\right)^{\prime} \geq\left(f_{e^{\prime}} d_{e^{\prime}}\left(f_{e^{\prime}}\right)\right)^{\prime} \geq\left(g_{e^{\prime}} d_{e^{\prime}}\left(g_{e^{\prime}}\right)\right)^{\prime}
$$

in the following. The first and last inequalities hold simply by the semiconvexity of $d_{e}$ and $d_{e^{\prime}}$. The second equality is obtained by setting $\xi=\frac{1-\alpha_{j}}{1-\alpha_{1}}$ to get $1-\xi=\alpha_{j}-\xi \alpha_{1}$, so Inequality (17) implies that

$$
\left(f_{e} d_{e}\left(f_{e}\right)\right)^{\prime}=d_{e}\left(f_{e}\right)+f_{e} d_{e}^{\prime}\left(f_{e}\right) \geq d_{e^{\prime}}\left(f_{e^{\prime}}\right)+f_{e^{\prime}} d_{e^{\prime}}^{\prime}\left(f_{e^{\prime}}\right)=\left(f_{e^{\prime}} d_{e^{\prime}}\left(f_{e^{\prime}}\right)\right)^{\prime} .
$$

Thus, $g$ can be made cheaper by moving some of its flow from $e$ to $e^{\prime}$. By repeating this process, we can thus assume that $g\left(E^{\prime}\right) \geq f\left(E^{\prime}\right)$.

Let $\gamma$ be such that $C\left(f-f^{\prime}\right)=\gamma C(\bar{f})$. Because $f^{\prime}$ and $f-f^{\prime}$ use disjoint sets of facilities, we get $C\left(f^{\prime}\right)=(1-\gamma) C(f)$. (Notice that the assumption of disjoint sets is indeed crucial. Due to the nonconstant cost of facilities, in general, it does not hold that $\left.C(f)+C\left(f^{\prime}\right)=C\left(f+f^{\prime}\right).\right)$

By Lemma 7.6, we can decompose $g=h+g^{\prime}$, where $g^{\prime}$ is a flow of rate $r^{\prime}$ entirely on $E^{\prime}$, and $h$ is a flow of rate $r_{1}$ satisfying the property (19), namely

$$
\tilde{C}(\tilde{f}) \leq \sum_{e} h_{e} d_{e}\left(g_{e}\right)+\sum_{e} g_{e}^{\prime}\left(d_{e}\left(g_{e}\right)-d_{e}\left(g_{e}^{\prime}\right)\right) .
$$

We can thus apply induction on the flows $f^{\prime}$ and $g^{\prime}$ of rate $r^{\prime}$ on the modified instance with facility set $E^{\prime}$. Notice that while $f^{\prime}$ may not be an equilibrium flow on $E$, it is indeed an equilibrium flow on $E^{\prime}$. Thus, we obtain that

$$
\begin{aligned}
C(g) & =C\left(g^{\prime}\right)+\sum_{e} h(e) d_{e}\left(g_{e}\right)+\sum_{e} g_{e}^{\prime}\left(d_{e}\left(g_{e}\right)-d_{e}\left(g_{e}^{\prime}\right)\right) \\
& \geq C\left(f^{\prime}\right) \sum_{i=2}^{k} \frac{r_{i}}{r^{\prime}} \frac{1}{\sigma^{\left(\alpha_{i}\right)}(\mathcal{C})}+C\left(f-f^{\prime}\right) \frac{1}{\sigma^{\left(\alpha_{1}\right)}(\mathcal{C})} \\
& =C(f) \sum_{i=2}^{k}\left(\frac{r_{i}}{r^{\prime}} \frac{1}{\sigma^{\left(\alpha_{i}\right)}(\mathcal{C})} \cdot(1-\gamma)+\frac{1}{\sigma^{\left(\alpha_{1}\right)}(\mathcal{C})} \cdot \gamma\right) .
\end{aligned}
$$

We next show that $\gamma \leq \frac{r_{1}}{r}$. By (16), every player on $E_{1}$ incurs lower delay than every player on $E_{j}$, and consequently on $E^{\prime}$. Thus, the average delay $\frac{1}{r_{1}} C\left(f-f^{\prime}\right)$ of players on $E_{1}$ is at most the average delay $\frac{1}{r} C(f)$ of all players, so $C\left(f-f^{\prime}\right) \leq \frac{r_{1}}{r} C(f)$.

The lower bound (18) is a convex combination of the nonnegative terms

$$
\sum_{i=2}^{k} \frac{r_{i}}{r^{\prime}} \frac{1}{\sigma^{\left(\alpha_{i}\right)}(\mathcal{C})} \quad \text { and } \quad \frac{1}{\sigma^{\left(\alpha_{1}\right)}(\mathcal{C})}
$$

with coefficients $(1-\gamma)$ and $\gamma$, respectively, The anarchy value $\sigma^{(\alpha)}(\mathcal{C})$ is a monotone nonincreasing function of $\alpha$, so the weighted average reciprocal anarchy value for 
altruism levels $\alpha_{2}, \ldots, \alpha_{k}$ is at least the reciprocal for $\alpha_{1}$. Thus, the convex combination is minimized when the coefficient $\gamma$ of the smaller term $\frac{1}{\sigma^{\left(\alpha_{1}\right)}(\mathcal{C})}$ is as large as possible, that is, when $\gamma=r_{1} / r$. Substituting this bound,

$$
C(g) \geq C(f) \sum_{i=2}^{k}\left(\frac{r_{i}}{r^{\prime}} \frac{1}{\sigma^{\left(\alpha_{i}\right)}(\mathcal{C})} \cdot \frac{r^{\prime}}{r}+\frac{1}{\sigma^{\left(\alpha_{1}\right)}(\mathcal{C})} \cdot \frac{r_{1}}{r}\right)=C(f) \sum_{i=1}^{k} \frac{r_{i}}{r^{\prime}} \frac{1}{\sigma^{\left(\alpha_{i}\right)}(\mathcal{C})},
$$

completing the inductive step, and thus the proof.

LEMMA 7.6. Let $f^{\prime}$ be a flow of rate $r^{\prime}$ using only facilities from $E^{\prime}$, and define $\tilde{d}_{e}(x):=$ $d_{e}\left(f_{e}^{\prime}+x\right)+\alpha_{1} f_{e}^{\prime} d_{e}^{\prime}\left(f_{e}^{\prime}\right)$. Let $g$ be any flow of rate $r=r^{\prime}+r_{1}$, with $g\left(E^{\prime}\right) \geq r^{\prime}$. Let $\tilde{f}$ be the optimum flow of rate $r_{1}$ with respect to facility costs $\tilde{d}_{e}$. Then, $g$ can be decomposed as $g=h+g^{\prime}$, where $g^{\prime}$ is a flow of rate $r^{\prime}$ on $E^{\prime}$, satisfying

$$
\tilde{C}(\tilde{f}) \leq \sum_{e} h_{e} d_{e}\left(g_{e}\right)+\sum_{e} g_{e}^{\prime}\left(d_{e}\left(g_{e}\right)-d_{e}\left(g_{e}^{\prime}\right)\right) .
$$

Proof. Let $\Delta:=g\left(E^{\prime}\right)-r^{\prime} \geq 0$ be the amount of "excess flow" that $g$ sends on $E^{\prime}$, compared to $f$. We begin by setting $h_{e}=g_{e}$ for all facilities $e \in E_{1}$, giving us a flow of rate $r_{1}-\Delta$. So we need to add $\Delta$ more units of flow to $h$. Let $E^{\prime \prime}:=\left\{e \in E^{\prime}: g_{e} \geq f_{e}^{\prime}\right\}$ be the set of facilities in $E^{\prime}$ on which $g$ sends more flow than $f^{\prime}$. Thus, we have that $g\left(E^{\prime \prime}\right)-f^{\prime}\left(E^{\prime \prime}\right) \geq g\left(E^{\prime}\right)-f^{\prime}\left(E^{\prime}\right)=\Delta$. In particular, we can define a flow $h$ of total rate $\Delta$ on $E^{\prime \prime}$, such that $h_{e} \leq g_{e}-f_{e}^{\prime}$ for all $e \in E^{\prime \prime}$. For all other facilities $e$, we set $h_{e}=0$, and thus obtain a flow $h$ of rate $r_{1}$, such that $h_{e} \leq g_{e}$ for all facilities $e$. We then have that

$$
\sum_{e} h_{e} d_{e}\left(g_{e}\right)=\sum_{e \in E_{1}} h_{e} d_{e}\left(h_{e}\right)+\sum_{e \in E^{\prime \prime}} h_{e} d_{e}\left(g_{e}\right) \geq \sum_{e \in E_{1}} h_{e} d_{e}\left(h_{e}\right)+\sum_{e \in E^{\prime \prime}} h_{e} d_{e}\left(f_{e}^{\prime}+h_{e}\right),
$$

where the inequality follows from the monotonicity of the delays $d_{e}$. Next, because $g_{e}^{\prime} \geq f_{e}^{\prime}$ for all $e \in E^{\prime}$, and the delay functions are convex, $\left(d_{e}\left(g_{e}\right)-d_{e}\left(g_{e}^{\prime}\right)\right) / h_{e} \geq d_{e}^{\prime}\left(f_{e}^{\prime}\right)$ for all $e \in E^{\prime \prime}$ with $h_{e}>0$. Combining this bound with the fact that $\alpha_{1} \leq 1$, we obtain that

$$
\sum_{e} g_{e}^{\prime}\left(d_{e}\left(g_{e}\right)-d_{e}\left(g_{e}^{\prime}\right)\right) \geq \sum_{e \in E^{\prime \prime}} g_{e}^{\prime}\left(d_{e}\left(g_{e}\right)-d_{e}\left(g_{e}^{\prime}\right)\right) \geq \sum_{e \in E^{\prime \prime}} f_{e}^{\prime} \alpha_{1} h_{e} d_{e}^{\prime}\left(f_{e}^{\prime}\right) .
$$

Summing the previous two inequalities now gives us

$$
\begin{aligned}
\sum_{e} h_{e} d_{e}\left(g_{e}\right)+\sum_{e} g_{e}^{\prime}\left(d_{e}\left(g_{e}\right)-d_{e}\left(g_{e}^{\prime}\right)\right) & \geq \sum_{e \in E_{1}} h_{e} d_{e}\left(h_{e}\right)+\sum_{e \in E^{\prime \prime}} h_{e} d_{e}\left(f_{e}^{\prime}+h_{e}\right)+\sum_{e \in E^{\prime \prime}} h_{e} \alpha_{1} f_{e}^{\prime} d_{e}^{\prime}\left(f_{e}^{\prime}\right) \\
& =\sum_{e} h_{e} \tilde{d}_{e}\left(h_{e}\right) \geq \tilde{C}(\tilde{f})
\end{aligned}
$$

where the final inequality follows from the optimality of $\tilde{f}$ with respect to the delay functions $\tilde{d}_{e}$.

\section{FAIR COST-SHARING GAMES}

In this section, we analyze the robust price of anarchy of fair cost-sharing games. It is well known that the pure price of anarchy is $n$ [Nisan et al. 2007]. We show that the pure price of anarchy can get significantly worse in the presence of altruistic players: the following theorem gives an upper bound of $n /(1-\hat{\alpha})$, which we subsequently show to be tight, even for the pure price of anarchy.

THEOREM 8.1. The robust price of anarchy of $\alpha$-altruistic cost-sharing games is at most $\frac{n}{1-\hat{\alpha}}($ with $n / 0=\infty)$. 
Proof. The claim is true for $\hat{\alpha}=1$ because $\operatorname{RPoA}(\alpha) \leq \infty$ holds trivially. We show that $G^{\alpha}$ is $(n, \hat{\alpha}, \boldsymbol{\alpha})$-smooth for $\hat{\alpha} \in[0,1)$. Let $\boldsymbol{s}$ and $\boldsymbol{s}^{\prime}$ be two strategy profiles. Fix an arbitrary player $i \in N$. We have

$$
C\left(s_{i}^{\prime}, \boldsymbol{s}_{-i}\right)-C(\boldsymbol{s})=\sum_{e \in U\left(s_{i}^{\prime}, \boldsymbol{s}_{-i}\right)} c_{e}-\sum_{e \in U(\boldsymbol{s})} c_{e} \leq \sum_{e \in s_{i}^{\prime} \backslash U(\boldsymbol{s})} c_{e} .
$$

We use this inequality to obtain the following bound:

$$
\begin{aligned}
\left(1-\alpha_{i}\right) C_{i}\left(s_{i}^{\prime}, \boldsymbol{s}_{-i}\right)+\alpha_{i}\left(C\left(s_{i}^{\prime}, \boldsymbol{s}_{-i}\right)-C(\boldsymbol{s})\right) & \leq\left(1-\alpha_{i}\right) \sum_{e \in s_{i}^{\prime}} \frac{c_{e}}{x_{e}\left(s_{i}^{\prime}, \boldsymbol{s}_{-i}\right)}+\alpha_{i} \sum_{e \in s_{i}^{\prime} \backslash U(\boldsymbol{s})} \frac{c_{e}}{x_{e}\left(s_{i}^{\prime}, \boldsymbol{s}_{-i}\right)} \\
& \leq \sum_{e \in s_{i}^{\prime}} \frac{c_{e}}{x_{e}\left(s_{i}^{\prime}, \boldsymbol{s}_{-i}\right)} \leq \sum_{e \in s_{i}^{\prime}} \frac{n \cdot c_{e}}{x_{e}\left(\boldsymbol{s}^{\prime}\right)} .
\end{aligned}
$$

The first inequality holds because $x_{e}\left(s_{i}^{\prime}, \boldsymbol{s}_{-i}\right)=1$ for every $e \in s_{i}^{\prime} \backslash U(\boldsymbol{s})$, and the last inequality follows from $x_{e}\left(s_{i}^{\prime}, \boldsymbol{s}_{-i}\right) \geq x_{e}\left(\boldsymbol{s}^{\prime}\right) / n$ for every $e \in s_{i}^{\prime}$. The left-hand side of the smoothness condition (5) can be rewritten as

$$
\begin{aligned}
\sum_{i=1}^{n}\left(\left(1-\alpha_{i}\right) C_{i}\left(s_{i}^{\prime}, \boldsymbol{s}_{-i}\right)+\alpha_{i}\left(C\left(s_{i}^{\prime}, \boldsymbol{s}_{-i}\right)-C(\boldsymbol{s})\right)+\alpha_{i} C_{i}(\boldsymbol{s})\right) & \leq \sum_{i=1}^{n}\left(\sum_{e \in s_{i}^{\prime}} \frac{n \cdot c_{e}}{x_{e}\left(\boldsymbol{s}^{\prime}\right)}\right)+\hat{\alpha} \boldsymbol{C}(\boldsymbol{s}) \\
& =n C\left(\boldsymbol{s}^{\prime}\right)+\hat{\alpha} C(\boldsymbol{s}) .
\end{aligned}
$$

We conclude that the robust price of anarchy is at most $\frac{n}{1-\hat{\alpha}}$. Example 8.2 shows that this bound is tight, even for pure Nash equilibria of symmetric singleton cost-sharing games.

Example 8.2. Consider the (symmetric singleton) cost-sharing game in which $n$ players can choose between two different facilities $e_{1}$ and $e_{2}$ of cost 1 and $n /(1-\alpha)$, respectively. Let $\boldsymbol{s}^{*}=\left(e_{1}, \ldots, e_{1}\right)$ and $\boldsymbol{s}=\left(e_{2}, \ldots, e_{2}\right)$ refer to the strategy profiles in which every player chooses $e_{1}$ and $e_{2}$, respectively. Then, $C\left(\boldsymbol{s}^{*}\right)=1$ and $C(\boldsymbol{s})=n /(1-\alpha)$. Note that $s$ is a pure Nash equilibrium of the $\alpha$-altruistic extension of this game because for every player $i$, we have

$$
(1-\alpha) C_{i}(\boldsymbol{s})+\alpha C(\boldsymbol{s})=1+\alpha \frac{n}{1-\alpha}=C_{i}^{\alpha}\left(\left\{e_{1}\right\}, \boldsymbol{s}_{-i}\right) .
$$

The pure price of anarchy is therefore at least $n /(1-\alpha)$.

Intuitively, the reason that the price of anarchy can get worse in the presence of altruism is that altruistic players worry more about hurting others with a strategy change, and are thus more likely to be stuck in a suboptimal equilibrium. The same phenomenon-being ready to accept more states as equilibria-should lead to a lower price of stability. Indeed, we next show an improved upper bound for the pure price of stability of uniformly $\alpha$-altruistic cost-sharing games. Note that for the completely selfish case $\alpha=0$, it is well known that the tight upper bound for the pure price of stability is $H_{n}$. Clearly, this upper bound extends to the mixed, correlated and coarse price of stability. The proof of the following proposition exploits a standard technique to bound the pure price of stability of exact potential games (see, e.g., Nisan et al. [2007]).

Proposition 8.3. The pure price of stability of uniformly $\alpha$-altruistic cost-sharing games is at most $(1-\alpha) H_{n}+\alpha$.

Proof. Let $G^{\alpha}$ be a uniformly $\alpha$-altruistic cost-sharing game. It is not hard to verify that $G^{\alpha}$ is an exact potential game with potential function $\Phi^{\alpha}(\boldsymbol{s})=(1-\alpha) \Phi(\boldsymbol{s})+\alpha C(\boldsymbol{s})$, 
where $\Phi(\boldsymbol{s})=\sum_{e \in E} \sum_{i=1}^{x_{e}(\boldsymbol{s})} c_{e} / i$. Observe that $\Phi^{\alpha}(\boldsymbol{s})=(1-\alpha) \sum_{e \in E} \sum_{i=1}^{x_{e}(\boldsymbol{s})} \frac{c_{e}}{i}+\alpha \sum_{e \in U(\boldsymbol{s})} c_{e} \leq\left((1-\alpha) H_{n}+\alpha\right) \sum_{e \in U(\boldsymbol{s})} c_{e}=\left((1-\alpha) H_{n}+\alpha\right) C(\boldsymbol{s})$.

We therefore have that $C(\boldsymbol{s}) \leq \Phi^{\alpha}(\boldsymbol{s}) \leq\left((1-\alpha) H_{n}+\alpha\right) C(\boldsymbol{s})$.

Let $s$ be a strategy profile that minimizes $\Phi^{\alpha}$, and let $\boldsymbol{s}^{*}$ be an optimal strategy profile that minimizes the social cost function $C$. Note that $s$ is a pure Nash equilibrium of $G^{\alpha}$. We have

$$
C(\boldsymbol{s}) \leq \Phi^{\alpha}(\boldsymbol{s}) \leq \Phi^{\alpha}\left(\boldsymbol{s}^{*}\right) \leq\left((1-\alpha) H_{n}+\alpha\right) C\left(\boldsymbol{s}^{*}\right),
$$

which proves the claim.

\section{VALID UTILITY GAMES}

In this section, we analyze valid utility games with altruism. Vetta [2002] proved a bound of 2 on the pure price of anarchy for valid utility games with nondecreasing $V$, and Roughgarden [2009] showed how this bound is achieved via a smoothness argument. We extend this result to altruistic extensions of these games.

THEOREM 9.1. The robust price of anarchy of $\alpha$-altruistic valid utility games is 2 .

Note that, in this statement the claim holds for arbitrary nonuniform altruism.

Proof. We show that the $\alpha$-altruistic extension $G^{\alpha}$ of a valid utility game is $(1,1, \alpha)$ smooth.

Fix two strategy profiles $\boldsymbol{s}, \boldsymbol{s}^{\prime} \in \Sigma$, and consider an arbitrary player $i \in N$. By definition of a valid utility game, we have

$$
\Pi_{i}(\boldsymbol{s}) \geq \Pi(\boldsymbol{s})-\Pi\left(\varnothing, \boldsymbol{s}_{-i}\right) .
$$

Therefore, for each player $i \in N$,

$$
\begin{aligned}
\Pi\left(s_{i}^{\prime}, \boldsymbol{s}_{-i}\right)-\Pi(\boldsymbol{s})+\Pi_{i}(\boldsymbol{s}) & =\left(\Pi\left(s_{i}^{\prime}, \boldsymbol{s}_{-i}\right)-\Pi\left(\varnothing, \boldsymbol{s}_{-i}\right)\right)-\left(\Pi(\boldsymbol{s})-\Pi\left(\varnothing, \boldsymbol{s}_{-i}\right)\right)+\Pi_{i}(\boldsymbol{s}) \\
& \geq \Pi\left(s_{i}^{\prime}, \boldsymbol{s}_{-i}\right)-\Pi\left(\varnothing, \boldsymbol{s}_{-i}\right) .
\end{aligned}
$$

Now let $U_{i}=\bigcup_{j=1}^{n} s_{j} \cup \bigcup_{j=1}^{i} s_{j}^{\prime}$. Recalling that $\Pi_{-i}(\boldsymbol{s})=\Pi(\boldsymbol{s})-\Pi_{i}(\boldsymbol{s})$, and summing over all $i \in N$,

$$
\begin{aligned}
& \sum_{i=1}^{n}\left(\left(1-\alpha_{i}\right) \Pi_{i}\left(s_{i}^{\prime}, \boldsymbol{s}_{-i}\right)+\alpha_{i}\left(\Pi\left(s_{i}^{\prime}, \boldsymbol{s}_{-i}\right)-\Pi(\boldsymbol{s})+\Pi_{i}(\boldsymbol{s})\right)\right) \\
& \quad \geq \sum_{i=1}^{n}\left(\Pi\left(s_{i}^{\prime}, \boldsymbol{s}_{-i}\right)-\Pi\left(\varnothing, \boldsymbol{s}_{-i}\right)\right) \geq \sum_{i=1}^{n}\left(V\left(U_{i}\right)-V\left(U_{i-1}\right)\right) \geq \Pi\left(\boldsymbol{s}^{\prime}\right)-\Pi(\boldsymbol{s}) .
\end{aligned}
$$

Here, the first inequality follows from (20) and (21), the second inequality holds because $V$ is submodular, and the final inequality follows from $V$ being nondecreasing. We conclude that $G^{\alpha}$ is $(1,1, \alpha)$-smooth, which proves an upper bound of 2 on the robust price of anarchy. This bound is tight, as shown by Example 9.2.

Example 9.2. Consider a valid utility game $G$ with two players $N=\{1,2\}$, a ground set $E=\{1,2\}$ of two elements and strategy sets $\Sigma_{1}=\{\{1\},\{2\}\}, \Sigma_{2}=\{\varnothing,\{1\}\}$. Define $V(S)=|S|$ for every subset $S \subseteq E$. Note that $V$ is nonnegative, nondecreasing, and submodular.

For a given strategy profile $s \in \Sigma$, the individual payoffs $\Pi_{1}(s)$ and $\Pi_{2}(s)$ of player 1 and player 2 , respectively, are defined as follows: $\Pi_{1}(s)=1$ for all strategy profiles $s$. 
$\Pi_{2}(s)=1$ if $s=(\{2\},\{1\})$ and $\Pi_{2}(s)=0$ otherwise. It is not hard to verify that for every player $i$ and every strategy profile $\boldsymbol{s} \in \Sigma$, we have $\Pi_{i}(\boldsymbol{s}) \geq \Pi(\boldsymbol{s})-\Pi\left(\varnothing, \boldsymbol{s}_{-i}\right)$. Moreover, $\Pi(\boldsymbol{s}) \geq \Pi_{1}(\boldsymbol{s})+\Pi_{2}(\boldsymbol{s})$ for every $\boldsymbol{s} \in \Sigma$. We conclude that $G$ is a valid utility game.

Let $\alpha \in[0,1]^{2}$ be arbitrary, and consider the $\alpha$-altruistic extension $G^{\alpha}$ of $G$. We claim that $s=(\{1\}, \varnothing)$ is a pure Nash equilibrium of $G^{\alpha}$. The utility of player 1 under $\boldsymbol{s}$ is $\left(1-\alpha_{1}\right)+\alpha_{1}=1$. His utility remains 1 if he switches to the strategy $\{2\}$. The utility of player 2 under $\boldsymbol{s}$ is $\alpha_{2}$. If he switches to the strategy $\{1\}$, then his utility is $\alpha_{2}$ as well. Thus, $\boldsymbol{s}$ is a pure Nash equilibrium. Since $\Pi(\boldsymbol{s})=1$ and $\Pi((\{2\},\{1\}))=2$, the pure price of anarchy of $G^{\alpha}$ is 2 .

\section{GENERAL PROPERTIES OF SMOOTHNESS}

Most of the analysis of games in this work (with the exception of symmetric singleton congestion games) proved robust price of anarchy results using $(\lambda, \mu, \alpha)$-smoothness as the key tool. In this section, we provide some general results about $(\lambda, \mu, \alpha)$-smoothness.

Proposition 10.1. Let $\mathcal{G}$ be a class of cost-minimization games equipped with sumbounded social cost functions. The set

$$
S_{\mathcal{G}}=\left\{(\lambda, \mu, \boldsymbol{\alpha}): G^{\alpha} \text { is }(\lambda, \mu, \boldsymbol{\alpha}) \text {-smooth for all } G \in \mathcal{G}\right\}
$$

is convex.

Proof. Let $G \in \mathcal{G}$ be arbitrary. It suffices to show that

$$
S_{G}=\left\{(\lambda, \mu, \boldsymbol{\alpha}): G^{\alpha} \text { is }(\lambda, \mu, \boldsymbol{\alpha}) \text {-smooth }\right\}
$$

is convex, because the intersection of any collection of convex sets is also convex.

Let $\left(\lambda_{1}, \mu_{1}, \boldsymbol{\alpha}^{(1)}\right),\left(\lambda_{2}, \mu_{2}, \boldsymbol{\alpha}^{(2)}\right) \in S_{G}$ be two elements in $S_{G}$, and let $\gamma \in[0,1]$ be arbitrary. For all pairs $\left(\boldsymbol{s}, \boldsymbol{s}^{\prime}\right)$ of strategy profiles of $G$, smoothness implies that

$$
\begin{aligned}
& \gamma \sum_{i=1}^{n}\left(C_{i}\left(s_{i}^{\prime}, \boldsymbol{s}_{-i}\right)+\alpha_{i}^{(1)}\left(C_{-i}\left(s_{i}^{\prime}, \boldsymbol{s}_{-i}\right)-C_{-i}(\boldsymbol{s})\right)\right) \\
& \quad+(1-\gamma) \sum_{i=1}^{n}\left(C_{i}\left(s_{i}^{\prime}, \boldsymbol{s}_{-i}\right)+\alpha_{i}^{(2)}\left(C_{-i}\left(s_{i}^{\prime}, \boldsymbol{s}_{-i}\right)-C_{-i}(\boldsymbol{s})\right)\right) \\
& \quad \leq \gamma\left(\lambda_{1} C\left(\boldsymbol{s}^{*}\right)+\mu_{1} C(\boldsymbol{s})\right)+(1-\gamma)\left(\lambda_{2} C\left(\boldsymbol{s}^{*}\right)+\mu_{2} C(\boldsymbol{s})\right) .
\end{aligned}
$$

By rewriting both sides of this inequality, we obtain

$$
\begin{gathered}
\sum_{i=1}^{n}\left(C_{i}\left(s_{i}^{\prime}, \boldsymbol{s}_{-i}\right)+\left(\gamma \alpha_{i}^{(1)}+(1-\gamma) \alpha_{i}^{(2)}\right) \cdot\left(C_{-i}\left(s_{i}^{\prime}, \boldsymbol{s}_{-i}\right)-C_{-i}(\boldsymbol{s})\right)\right) \\
\leq\left(\gamma \lambda_{1}+(1-\gamma) \lambda_{2}\right) C\left(\boldsymbol{s}^{*}\right)+\left(\gamma \mu_{1}+(1-\gamma) \mu_{2}\right) C(\boldsymbol{s}) .
\end{gathered}
$$

We conclude that $G$ is $\left(\gamma\left(\lambda_{1}, \mu_{1}, \boldsymbol{\alpha}^{(1)}\right)+(1-\gamma)\left(\lambda_{2}, \mu_{2}, \boldsymbol{\alpha}^{(2)}\right)\right)$-smooth. Therefore, $S_{G}$ is convex.

A natural question to ask is whether the robust price of anarchy is also a convex function of $\alpha$. This turns out not to be the case. For instance, the robust price of anarchy for uniformly $\alpha$-altruistic congestion games is $\frac{5+4 \alpha}{2+\alpha}$ (see Section 4 ), which is a non-convex function. However, we can prove a somewhat weaker statement: For a subset $S \subseteq \mathbb{R}^{n}$, we call a function $f: S \rightarrow \mathbb{R}$ quasi-convex iff $f(\gamma x+(1-\gamma) y) \leq \max (f(x), f(y))$ for all $\gamma \in[0,1]$.

Theorem 10.2. Let $\mathcal{G}$ be a class of games equipped with sum-bounded social cost functions. Then, $R P o A_{\mathcal{G}}(\boldsymbol{\alpha})$ is a quasiconvex function of $\boldsymbol{\alpha}$. 
Proof. Let $G \in \mathcal{G}$. We show that for any $\boldsymbol{\alpha}^{(1)}, \boldsymbol{\alpha}^{(2)} \in \mathbb{R}^{n}$ and $\gamma \in[0,1]$,

$$
\operatorname{RPoA}\left(\gamma \boldsymbol{\alpha}^{(1)}+(1-\gamma) \boldsymbol{\alpha}^{(2)}\right) \leq \max \left(\operatorname{RPoA}\left(\boldsymbol{\alpha}^{(1)}\right), \operatorname{RPoA}\left(\boldsymbol{\alpha}^{(2)}\right)\right) .
$$

Let $\epsilon_{1}, \epsilon_{2}, \ldots$ be a decreasing sequence of positive real numbers that tends to 0 . Moreover, let $\left(\lambda_{1,1}, \mu_{1,1}, \boldsymbol{\alpha}^{(1)}\right),\left(\lambda_{1,2}, \mu_{1,2}, \boldsymbol{\alpha}^{(1)}\right), \ldots$ and $\left(\lambda_{2,1}, \mu_{2,1}, \boldsymbol{\alpha}^{(2)}\right),\left(\lambda_{2,2}, \mu_{2,2}, \boldsymbol{\alpha}^{(2)}\right), \ldots$ be sequences of elements in $S_{G}$ (where $S_{G}$ is as defined in the proof of Proposition 10.1) such that for all $j$,

$$
\operatorname{RPoA}\left(\boldsymbol{\alpha}^{(1)}\right)+\epsilon_{j}=\frac{\lambda_{1, j}}{1-\mu_{1, j}} \quad \text { and } \quad \operatorname{RPoA}\left(\boldsymbol{\alpha}^{(2)}\right)+\epsilon_{j}=\frac{\lambda_{2, j}}{1-\mu_{2, j}} .
$$

By Proposition 10.1, for all $j$,

$$
\begin{aligned}
& \sum_{i=1}^{n}\left(C_{i}\left(s_{i}^{\prime}, \boldsymbol{s}_{-i}\right)+\left(\gamma \alpha_{i}^{(1)}+(1-\gamma) \alpha_{i}^{(2)}\right) \cdot\left(C_{-i}\left(s_{i}^{\prime}, \boldsymbol{s}_{-i}\right)-C_{-i}(\boldsymbol{s})\right)\right) \\
& \quad \leq \gamma\left(\lambda_{1, j} C\left(\boldsymbol{s}^{\prime}\right)+\mu_{1, j} C(\boldsymbol{s})\right)+(1-\gamma)\left(\lambda_{2, j} C\left(\boldsymbol{s}^{\prime}\right)+\mu_{2, j} C(\boldsymbol{s})\right) \\
& \quad \leq \max \left(\lambda_{1, j} C\left(\boldsymbol{s}^{\prime}\right)+\mu_{1, j} C(\boldsymbol{s}), \lambda_{2, j} C\left(\boldsymbol{s}^{\prime}\right)+\mu_{2, j} C(\boldsymbol{s})\right) .
\end{aligned}
$$

Hence, for all $j$,

$$
\begin{aligned}
\operatorname{RPoA}\left(\gamma \boldsymbol{\alpha}^{(1)}+(1-\gamma) \boldsymbol{\alpha}^{(2)}\right) & \leq \max \left(\frac{\lambda_{1, j}}{1-\mu_{1, j}}, \frac{\lambda_{2, j}}{1-\mu_{2, j}}\right) \\
& \leq \max \left(\operatorname{RPoA}\left(\boldsymbol{\alpha}^{(1)}\right), \operatorname{RPoA}\left(\boldsymbol{\alpha}^{(2)}\right)\right)+\epsilon_{j}
\end{aligned}
$$

By taking the limit $j \rightarrow \infty$, we conclude $\operatorname{RPoA}\left(\gamma \boldsymbol{\alpha}^{(1)}+(1-\gamma) \boldsymbol{\alpha}^{(2)}\right) \leq$ $\max \left(\operatorname{RPoA}\left(\boldsymbol{\alpha}^{(1)}\right), \operatorname{RPoA}\left(\boldsymbol{\alpha}^{(2)}\right)\right)$, which proves the claim.

The quasiconvexity of $\mathrm{RPoA}_{\mathcal{G}}$ implies:

CoRollary 10.3. The altruism vectors $\boldsymbol{\alpha}$ that minimize $R P o A_{\mathcal{G}}(\boldsymbol{\alpha})$ on the domain $[0,1]^{n}$ form a convex set. The altruism vectors $\boldsymbol{\alpha}$ that maximize $R P o A_{\mathcal{G}}(\boldsymbol{\alpha})$ on the domain $[0,1]^{n}$ include at least one point that is a 0-1 vector.

\section{CONCLUSIONS AND FUTURE WORK}

At first sight, it might seem counterintuitive that the price of anarchy is greater than 1 when every player is entirely altruistic, that is, $\boldsymbol{\alpha}=\mathbf{1}$. This phenomenon is less surprising when viewed from a local-search perspective. Note that for $\alpha=\mathbf{1}$, all players seek to minimize the social cost function $C$. A pure Nash equilibrium then corresponds to a local optimum of $C$ with respect to the neighborhood of single-player deviations. Our bounds on the pure price of anarchy therefore also bound the relative gap between the worst-case cost of a local optimum and the cost of a global optimum; this gap is also known as locality gap and was introduced by Arya et al. [2004].

The phenomenon that the price of anarchy can get worse as the altruism level $\alpha$ gets closer to 1 has been observed before (see Caragiannis et al. [2010]). The fact that the price of anarchy does not necessarily get worse in all cases is exemplified by our analysis of the price of anarchy in nonatomic congestion games and the pure price of anarchy in symmetric singleton congestion games.

The most immediate future directions include analyzing atomic singleton congestion games with more general delay functions than linear ones. While the price of anarchy of such functions increases (e.g., the price of anarchy for polynomials increases exponentially in the degree [Awerbuch et al. 2005; Christodoulou and Koutsoupias 2005]), this also creates room for potentially larger reductions due to altruism. Similarly, the 
characterization of the robust price of anarchy of atomic altruistic congestion games with more general delay functions (e.g., polynomials) is left for future work.

For games where the smoothness argument cannot give tight bounds, would a refined smoothness argument like local smoothness in Roughgarden and Schoppmann [2011] work? For symmetric singleton congestion games, this seems unlikely, as the price of anarchy of pure and mixed Nash equilibria differ. It is also worth trying to apply the smoothness argument or its refinements to analyze the price of anarchy for other dynamics in other classes of altruistic games, for example, (altruistic) network vaccination games [Chen et al. 2010], which are known to not always possess pure Nash equilibria, or to find examples to see why smoothness-based arguments do not work.

Our work also suggests several interesting directions for further research regarding non-atomic congestion games. First, how far can the analysis with arbitrary altruism distributions be extended? For network congestion games with arbitrary topologies (and just one type of player), any improved result would prove the corresponding bounds on Stackelberg routing, so the lower bound of Bonifaci et al. [2007] precludes a general extension. However, an extension to restricted classes of network congestion games (e.g., series-parallel graphs) seems plausible at this point. While we proved the existence of Nash equilibria for all routing games with non-atomic players, regardless of the distributions of altruism, the proof is nonconstructive. The work of Roughgarden [2004] implies that finding the best Stackelberg strategy (and thus the best equilibrium with altruism levels in $\{0,1\})$ is NP-complete. However, it would be interesting whether other equilibria can always be found efficiently. Alternatively, in light of the PPADcompleteness of finding Nash equilibria [Chen and Deng 2006], it may be possible that finding Nash equilibria for network congestion games with two (or more) altruism values is also PPAD-complete.

We have seen that the impact of altruism depends on the underlying game. It would be nice to identify general properties that enable one to predict whether a given game suffers from altruism or not. What is it that makes valid utility games invariant to altruism? Furthermore, what kind of "transformations" (not just altruistic extensions) might be applied to a strategic game such that the smoothness approach can still be adapted to give (tight) bounds? More generally, while the existence of pure Nash equilibria has been shown for singleton and matroid congestion games with player-specific delay functions [Ackermann et al. 2006; Milchtaich 1996], the price of anarchy (for pure Nash equilibria or more general equilibrium concepts) has not yet been addressed. Studying the price of anarchy in such a general setting (in which our setting with altruism can be embedded) by either smoothness-based techniques or other methods is undoubtedly intriguing.

\section{APPENDIXES}

\section{A. PROOFS FOR SECTION 3.3}

Proposition 3.5. Let $G^{\alpha}$ be an $\alpha$-altruistic extension of a game with sum-bounded social cost function $C$. If $G^{\alpha}$ is $(\lambda, \mu, \alpha)$-smooth with $\mu<1$, then the coarse (and thus correlated, mixed, and pure) price of anarchy of $G^{\alpha}$ is at most $\frac{\lambda}{1-\mu}$.

Proof. Let $\sigma$ be a coarse equilibrium of $G^{\alpha}, \boldsymbol{s}$ a random strategy profile with joint distribution $\sigma$, and $\boldsymbol{s}^{*} \in \Sigma$ a socially optimal strategy profile. The coarse equilibrium condition implies that for every player $i \in N$ :

$$
\mathbf{E}\left[\left(1-\alpha_{i}\right) C_{i}(\boldsymbol{s})+\alpha_{i} C(\boldsymbol{s})\right] \leq \mathbf{E}\left[\left(1-\alpha_{i}\right) C_{i}\left(s_{i}^{*}, \boldsymbol{s}_{-i}\right)+\alpha_{i} C\left(s_{i}^{*}, \boldsymbol{s}_{-i}\right)\right] .
$$


By linearity of expectation, for every player $i \in N$ :

$$
\mathbf{E}\left[C_{i}(\boldsymbol{s})\right] \leq \mathbf{E}\left[C_{i}\left(s_{i}^{*}, \boldsymbol{s}_{-i}\right)+\alpha_{i}\left(C\left(s_{i}^{*}, \boldsymbol{s}_{-i}\right)-C_{i}\left(s_{i}^{*}, \boldsymbol{s}_{-i}\right)\right)-\alpha_{i}\left(C(\boldsymbol{s})-C_{i}(\boldsymbol{s})\right)\right] .
$$

By summing over all players, using linearity of expectation and the sum-boundedness of $C$, we obtain

$$
\mathbf{E}[C(\boldsymbol{s})] \leq \mathbf{E}\left[\sum_{i=1}^{n} C_{i}\left(s_{i}^{*}, \boldsymbol{s}_{-i}\right)+\alpha_{i}\left(C_{-i}\left(s_{i}^{*}, \boldsymbol{s}_{-i}\right)-C_{-i}(\boldsymbol{s})\right)\right] .
$$

Now we use the smoothness property (5) to conclude

$$
\mathbf{E}[C(\boldsymbol{s})] \leq \mathbf{E}\left[\lambda C\left(\boldsymbol{s}^{*}\right)+\mu C(\boldsymbol{s})\right]=\lambda C\left(\boldsymbol{s}^{*}\right)+\mu \mathbf{E}[C(\boldsymbol{s})] .
$$

Solving for $\mathbf{E}[C(s)]$ and exploiting that $\mu<1$, now proves the claim. As coarse equilibria include correlated equilibria, mixed Nash equilibria and pure Nash equilibria, the correlated, mixed, and pure price of anarchy are thus also bounded by $\frac{\lambda}{1-\mu}$.

Proposition 3.6. Let $G^{\alpha}$ be a uniformly $\alpha$-altruistic nonatomic congestion game. If $G^{\alpha}$ is $(\lambda, \mu, \alpha)$-smooth with $\mu<1$, then the coarse price of anarchy of $G^{\alpha}$ is at most $\frac{\lambda}{1-\mu}$.

Proof. Let $F$ be a coarse equilibrium of the game $G^{\alpha}$. Thus, $F$ is a distribution over feasible flows $f$. Let $f^{*}$ be a feasible flow minimizing $C$. Consider a player type $\mathcal{S}$ and an infinitesimally small player of type $\mathcal{S} . F$ induces a distribution $F_{\mathcal{S}}$ over flows $f_{\mathcal{S}}$ for players of type $\mathcal{S}$, and thus also over strategies $s \in \mathcal{S}$ for those players. The distribution satisfies $\operatorname{Prob}_{f_{\mathcal{S}} \sim F_{\mathcal{S}}}\left[s=S \mid f_{\mathcal{S}}\right]=f_{\mathcal{S}, S} / r_{\mathcal{S}}$. The expected cost of a player of type $\mathcal{S}$ under $F$ is thus

$$
\begin{aligned}
\mathbf{E}_{(s, f) \sim F}\left[\sum_{e \in S} d_{e}\left(f_{e}\right)+\alpha f_{e} d_{e}^{\prime}\left(f_{e}\right)\right] & =\mathbf{E}_{f \sim F}\left[\sum_{S \in \mathcal{S}} \frac{f_{\mathcal{S}, S}}{r_{\mathcal{S}}} \cdot \sum_{e \in S} d_{e}\left(f_{e}\right)+\alpha f_{e} d_{e}^{\prime}\left(f_{e}\right)\right] \\
& =\frac{1}{r_{\mathcal{S}}} \cdot \sum_{S \in \mathcal{S}} \mathbf{E}_{f \sim F}\left[f_{\mathcal{S}, S} \cdot \sum_{e \in S} d_{e}\left(f_{e}\right)+\alpha f_{e} d_{e}^{\prime}\left(f_{e}\right)\right],
\end{aligned}
$$

by linearity of expectation. Because $F$ is a coarse equilibrium, it also satisfies

$$
\mathbf{E}_{(s, f) \sim F}\left[\sum_{e \in s} d_{e}\left(f_{e}\right)+\alpha f_{e} d_{e}^{\prime}\left(f_{e}\right)\right] \leq \mathbf{E}_{f \sim F}\left[\sum_{e \in s^{\prime}} d_{e}\left(f_{e}\right)+\alpha f_{e} d_{e}^{\prime}\left(f_{e}\right)\right]
$$

for every $s^{\prime} \in \mathcal{S}$.

For each $s^{\prime} \in \mathcal{S}$, we multiply (24) by $f_{\mathcal{S}, s^{\prime}}^{*}$; adding these inequalities for all $s^{\prime} \in \mathcal{S}$; substituting Eq. (23) and using that $\sum_{s^{\prime} \in \mathcal{S}} f_{\mathcal{S}, s^{\prime}}^{*}=r_{\mathcal{S}}$, then implies that

$$
\sum_{S \in \mathcal{S}} \mathbf{E}_{f \sim F}\left[f_{\mathcal{S}, S} \cdot \sum_{e \in S} d_{e}\left(f_{e}\right)+\alpha f_{e} d_{e}^{\prime}\left(f_{e}\right)\right] \leq \sum_{S \in \mathcal{S}} f_{\mathcal{S}, S}^{*} \cdot \mathbf{E}_{f \sim F}\left[\sum_{e \in S} d_{e}\left(f_{e}\right)+\alpha f_{e} d_{e}^{\prime}\left(f_{e}\right)\right] .
$$

Summing over all types $\mathcal{S}$, using linearity of expectation, and changing the order of summation between $(\mathcal{S}, S)$ and $e$ then gives

$$
\mathbf{E}_{f \sim F}\left[\sum_{e} f_{e} d_{e}\left(f_{e}\right)+\alpha f_{e}^{2} d_{e}^{\prime}\left(f_{e}\right)\right] \leq \mathbf{E}_{f \sim F}\left[\sum_{e} f_{e}^{*} d_{e}\left(f_{e}\right)+\alpha f_{e}^{*} f_{e} d_{e}^{\prime}\left(f_{e}\right)\right],
$$


which can be rearranged to give

$$
\mathbf{E}_{f \sim F}[C(f)]=\mathbf{E}_{f \sim F}\left[\sum_{e} f_{e} d_{e}\left(f_{e}\right)\right] \leq \mathbf{E}_{f \sim F}\left[\sum_{e} f_{e}^{*} d_{e}\left(f_{e}\right)+\alpha\left(f_{e}^{*}-f_{e}\right) f_{e} d_{e}^{\prime}\left(f_{e}\right)\right] .
$$

By the definition of smoothness for nonatomic games (see Eq. (6)), the right-hand side is upper-bounded by

$$
\mathbf{E}_{f \sim F}\left[\lambda C\left(f^{*}\right)+\mu C(f)\right]=\lambda C\left(f^{*}\right)+\mu \mathbf{E}_{f \sim F}[C(f)] .
$$

Now, solving for $\mathbf{E}_{f \sim F}[C(f)]$ shows that $\mathbf{E}_{f \sim F}[C(f)] \leq \frac{\lambda}{1-\mu} \cdot C\left(f^{*}\right)$.

Proposition 3.8. Let $G^{\alpha}$ be an $\alpha$-altruistic extension of a game with sum-bounded social cost function $C$. Let $\boldsymbol{s}^{*}$ be a strategy profile minimizing the social cost function $C$ of $G^{\alpha}$, and let $\boldsymbol{s}^{1}, \ldots, \boldsymbol{s}^{T}$ be a sequence of strategy profiles in which every player $i \in N$ experiences vanishing average external regret, that is,

$$
\sum_{t=1}^{T} C_{i}^{\alpha}\left(\boldsymbol{s}^{t}\right) \leq\left(\min _{s_{i}^{\prime} \in \Sigma_{i}} \sum_{t=1}^{T} C_{i}^{\alpha}\left(s_{i}^{\prime}, \boldsymbol{s}_{-i}^{t}\right)\right)+o(T) .
$$

The average cost of this sequence of $T$ strategy profiles then satisfies

$$
\frac{1}{T} \sum_{t=1}^{T} C\left(s^{t}\right) \leq R P o A(\alpha) \cdot C\left(s^{*}\right) \quad \text { as } T \rightarrow \infty .
$$

Proof. Consider a sequence $\boldsymbol{s}^{1}, \ldots, \boldsymbol{s}^{T}$ of strategy profiles of an $\alpha$-altruistic game $G^{\alpha}$ that is $(\lambda, \mu, \alpha)$-smooth with $\mu<1$. For every $i \in N$ and $t \in\{1, \ldots, T\}$, define

$$
\delta_{i, t}=C_{i}^{\alpha}\left(\boldsymbol{s}^{t}\right)-C_{i}^{\alpha}\left(s_{i}^{*}, \boldsymbol{s}_{-i}^{t}\right) .
$$

Let $\Delta_{t}=\sum_{i=1}^{n} \delta_{i, t}$. We have

$$
\begin{aligned}
\Delta_{t} & =\sum_{i=1}^{n} C_{i}^{\alpha}\left(\boldsymbol{s}^{t}\right)-C_{i}^{\alpha}\left(s_{i}^{*}, \boldsymbol{s}_{-i}^{t}\right) \\
& =\sum_{i=1}^{n}\left(\left(1-\alpha_{i}\right) C_{i}\left(\boldsymbol{s}^{t}\right)+\alpha_{i} C\left(\boldsymbol{s}^{t}\right)-\left(\left(1-\alpha_{i}\right) C_{i}\left(s_{i}^{*}, \boldsymbol{s}_{-i}^{t}\right)+\alpha_{i} C\left(s_{i}^{*}, \boldsymbol{s}_{-i}^{t}\right)\right)\right) \\
& \geq C\left(\boldsymbol{s}^{t}\right)-\sum_{i=1}^{n}\left(C_{i}\left(s_{i}^{*}, \boldsymbol{s}_{-i}^{t}\right)+\alpha_{i}\left(C_{-i}\left(s_{i}^{*}, \boldsymbol{s}_{-i}^{t}\right)-C_{-i}\left(\boldsymbol{s}^{t}\right)\right)\right)
\end{aligned}
$$

where the inequality holds because $C$ is sum-bounded.

Exploiting the $(\lambda, \mu, \alpha)$-smoothness property, we obtain

$$
C\left(s^{t}\right) \leq \frac{\lambda}{1-\mu} C\left(s^{*}\right)+\frac{1}{1-\mu} \Delta_{t} .
$$

By assumption, $\boldsymbol{s}^{1}, \ldots, \boldsymbol{s}^{T}$ is a sequence of strategy profiles in which every player experiences vanishing average external regret, that is, for every $i \in N$, Inequality (8) holds. We obtain that for every player $i \in N$ :

$$
\frac{1}{T} \sum_{t=1}^{T} \delta_{i, t} \leq \frac{1}{T}\left(\sum_{t=1}^{T} C_{i}^{\alpha}\left(\boldsymbol{s}^{t}\right)-\min _{s_{i}^{\prime} \in \Sigma_{i}} \sum_{t=1}^{T} C_{i}^{\alpha}\left(s_{i}^{\prime}, \boldsymbol{s}_{-i}^{t}\right)\right)=o(1) .
$$


Using this inequality and (25), we obtain that the average cost of the sequence of $T$ strategy profiles is

$$
\frac{1}{T} \sum_{t=1}^{T} C\left(\boldsymbol{s}^{t}\right) \leq \frac{\lambda}{1-\mu} C\left(\boldsymbol{s}^{*}\right)+\frac{1}{1-\mu} \sum_{i=1}^{n}\left(\frac{1}{T} \sum_{t=1}^{T} \delta_{i, t}\right) \stackrel{T \rightarrow \infty}{\longrightarrow} \frac{\lambda}{1-\mu} C\left(\boldsymbol{s}^{*}\right) .
$$

Proposition 3.9. Consider a uniformly $\alpha$-altruistic nonatomic congestion game $G^{\alpha}$ that is $(\lambda, \mu, \alpha)$-smooth with $\mu<1$. Let $f^{*}$ be a flow minimizing the social cost $C$, and $f^{1}, \ldots, f^{T}$ a sequence of flows in which every infinitesimal player $y \in[0, r]$ experiences vanishing average external regret in the following sense:

$$
\sum_{t=1}^{T} \sum_{e \in \phi^{t}(y)} d_{e}\left(f_{e}^{t}\right)+\alpha f_{e}^{t} d_{e}^{\prime}\left(f_{e}^{t}\right) \leq \min _{\hat{S} \in \tau(y)} \sum_{t=1}^{T} \sum_{e \in \hat{S}}\left(d_{e}\left(f_{e}^{t}\right)+\alpha f_{e}^{t} d_{e}^{\prime}\left(f_{e}^{t}\right)\right)+o(T) .
$$

The average cost of this sequence of $T$ flows then satisfies

$$
\frac{1}{T} \sum_{t=1}^{T} C\left(f^{t}\right) \leq R P o A(\alpha) \cdot C\left(f^{*}\right)+o(1)
$$

as $T \rightarrow \infty$.

Proof. Consider a sequence $f^{1}, \ldots, f^{T}$ of flows with vanishing average external regret in the sense defined here. Let $\phi^{t}$ be the corresponding mappings of strategies; similarly, let $\phi^{*}$ be a mapping from $[0, r]$ corresponding to the optimum flow $f^{*}$. For every player $y \in[0, r]$ and $t \in\{1, \ldots, T\}$, define the difference in cost between $y$ 's strategy at time $t$ and the cost $y$ would incur by unilaterally choosing the strategy $\phi^{*}(y)$ :

$$
\delta_{y, t}=\sum_{e \in \phi^{t}(y)}\left(d_{e}\left(f_{e}^{t}\right)+\alpha f_{e}^{t} d_{e}^{\prime}\left(f_{e}^{t}\right)\right)-\sum_{e \in \phi^{*}(y)}\left(d_{e}\left(f_{e}^{t}\right)+\alpha f_{e}^{t} d_{e}^{\prime}\left(f_{e}^{t}\right)\right) .
$$

Applying the vanishing regret condition to player $y$, we obtain that $\frac{1}{T} \sum_{t=1}^{T} \delta_{y, t} \leq o(1)$.

Let $\Delta_{t}=\int_{0}^{r} \delta_{y, t} d y$. By exchanging the integration and summation,

$$
\Delta_{t}\left(f^{t}\right)=\sum_{e \in E} f_{e}^{t} d_{e}\left(f_{e}^{t}\right)-\sum_{e \in E}\left(f_{e}^{*} d_{e}\left(f_{e}^{t}\right)+\left(f_{e}^{*}-f_{e}^{t}\right) \alpha f_{e}^{t} d_{e}^{\prime}\left(f_{e}^{t}\right)\right) \geq C\left(f^{t}\right)-\left(\lambda C\left(f^{*}\right)+\mu C\left(f^{t}\right)\right),
$$

by the $(\lambda, \mu, \alpha)$-smoothness property (6). Solving for $C\left(f^{t}\right)$, we obtain

$$
C\left(f^{t}\right) \leq \frac{\lambda}{1-\mu} C\left(f^{*}\right)+\frac{1}{1-\mu} \Delta\left(f^{t}\right) .
$$

Combining this bound with the vanishing regret condition, the average cost of the sequence of $T$ flows is

$$
\frac{1}{T} \sum_{t=1}^{T} C\left(f^{t}\right) \leq \frac{\lambda}{1-\mu} \cdot C\left(f^{*}\right)+\frac{1}{1-\mu} \cdot \frac{1}{T} \cdot \sum_{t=1}^{T} \delta_{y, t} \rightarrow \frac{\lambda}{1-\mu} \cdot C\left(f^{*}\right),
$$

as $T \rightarrow \infty$. 


\section{B. PROOFS FOR SECTION 4}

Lemma 4.3. Without loss of generality, all delay functions are of the form $d_{e}(x)=x$.

Proof. First, we may assume that for every delay function $d_{e}$, the $a_{e}$ and $b_{e}$ coefficients are integers. This can be ensured by multiplying all coefficients among all facilities by the least common multiple of all denominators. In the resulting game, all coefficients are integers, the price of anarchy is the same, and so is the set of all equilibria.

Next, we can assume that $b_{e}=0$ for all $e \in E$. To show this, we replace any facility $e \in E$ with delay function $d(x)=a_{e} x+b_{e}$ by $n+1$ facilities $e_{0}, \ldots, e_{n}$ with delay functions $d_{e_{0}}(x)=a_{e} x$ and $d_{e_{j}}(x)=b_{e} x$ for $1 \leq j \leq n$. We then adapt the strategy space $\Sigma_{i}$ of each player $i$ as follows: we replace every strategy $s_{i} \in \Sigma_{i}$ in which $e$ occurs by the strategy $s_{i} \backslash\{e\} \cup\left\{e_{0}, e_{i}\right\}$. There is an obvious bijection between the strategy profiles in the original game and those in the new game, preserving the values of individual cost functions and the social cost function. (Notice that this construction exploits the fact that all players have unit weight, and would not carry over to weighted congestion games.)

Finally, for the same reason, we can also assume that $a_{e}=1$ for all $e \in E$. We replace $e$ with facilities $e_{1}, \ldots, e_{a_{e}}$, each having delay function $d_{e_{i}}(x)=x$, and adapt the strategy space $\Sigma_{i}$ of each player $i$ by replacing each strategy $s_{i}$ in which $e$ occurs by $s_{i} \backslash\{e\} \cup\left\{e_{1}, \ldots, a_{a_{e}}\right\}$. Now, all delay functions are $d_{e}(x)=x$.

Lemma 4.5. For all $x, y \in \mathbb{N}_{0}, \eta \in[0,1]$ and $\beta \in[0,1]$, and all $\gamma \in\left[\frac{1}{3}(1+\eta-2 \beta \eta), 1+\eta\right]$,

$$
((1+\eta) x+1) y+\beta \eta(1-x) x \leq(2+\eta-\gamma) y^{2}+\gamma x^{2} .
$$

Proof. The inequality is equivalent to

$$
((1+\eta) x+1) y+\beta \eta(1-x) x-(2+\eta) y^{2} \leq \gamma\left(x^{2}-y^{2}\right) .
$$

Assume that $x=y$. The inequality is then trivially satisfied because $x \leq x^{2}$ for all $x \in \mathbb{N}_{0}$. Next, suppose that $x>y$. Then, we need to show that for all $\gamma$ in the given range,

$$
\gamma \geq \frac{((1+\eta) x+1) y+\beta \eta(1-x) x-(2+\eta) y^{2}}{x^{2}-y^{2}} .
$$

We show that the maximum of the expression on the right-hand side is attained by $x=2$ and $y=1$. First, we fill in these values: the right-hand side evaluates to $\frac{1}{3}(1+\eta-2 \beta \eta) \geq 0$, and $\gamma \geq \frac{1}{3}(1+\eta-2 \beta \eta)$ by assumption on $\gamma$. We now write $x$ as $y+a, a \geq 1$, and rewrite the right-hand side as

$$
f(y, a)=\frac{(1+\eta) y+\beta \eta}{2 y+a}+\frac{(1+\beta \eta)\left(y-y^{2}\right)}{a(2 y+a)}-\beta \eta .
$$

Because we know that there are choices of $y$ and $a$ for which $f(y, a)$ is positive (e.g., when $y=1$ and $a=1$ ), and because $a$ only occurs in the denominators, $f(y, a)$ reaches its maximum when $a=1$. So we assume that $a=1$. When we then set $y=0$, we see that $f(0,1)=0$, so $f(1,1) \geq f(0,1)$. When $y>1$, we can write $y$ as $w+2$, where $w \geq 0$; we can now further rewrite $f(y, a)$ as

$$
f(w+2,1)=\frac{2 \eta-6 \beta \eta}{2 w+5}-\frac{(2-\eta+5 \beta \eta) w+(1+\beta \eta) w^{2}}{2 w+5} \leq \frac{2 \eta-6 \beta \eta}{2 w+5} .
$$

When $2 \eta-6 \beta \eta$ is negative, this term is certainly less than $f(1,1)$. When $2 \eta-6 \beta \eta$ is positive, we have

$$
f(w+2,1) \leq \frac{2 \eta-6 \beta \eta}{2 w+5} \leq \frac{2 \eta-6 \beta \eta}{5} \leq \frac{1}{3}(2 \eta-6 \beta \eta) \leq \frac{1}{3}(1+\eta-2 \beta \eta)=f(1,1) .
$$


Thus, $f(y, a) \leq f(1,1)=\frac{1}{3}(1+\eta-2 \beta \eta)$; in particular, the inequality is always satisfied when $\gamma \leq \frac{1}{3}(1+\eta-2 \beta \eta)$.

The final case is when $x<y$. Then, the inequality we need to prove is equivalent to

$$
\gamma \leq \frac{(2+\eta) y^{2}-((1+\eta) x+1) y-\beta \eta(1-x) x}{y^{2}-x^{2}} .
$$

We show that the minimum of the expression on the right-hand side is attained by $x=0$ and $y=1$. First, we fill in these values: the right-hand side then evaluates to $1+\eta$, so for all choices of $\gamma$, we do indeed have $\gamma \leq 1+\eta$. We now write $y=x+a, a \geq 1$, and rewrite the right-hand side as

$$
g(x, a)=\frac{(1+\beta \eta) x^{2}-(1+a+(a+\beta) \eta) x-a}{a(2 x+a)}+2+\eta .
$$

Suppose first that $x=0$ and that $a \geq 2$. Then, we can write $a$ as $1+b, b>0$, and therefore

$$
g(0,1+b)=2+\eta-\frac{1}{1+b} \geq \frac{3}{2}+\eta \geq 1+\eta=g(0,1) .
$$

When $x \geq 1$, we can write $x=b+1, b \geq 0$. We then have

$$
g(1+b, a)=2+\eta-\frac{2+\eta+(1-\eta) b}{2 b+2+a}+\frac{(1+\beta \eta)\left(b^{2}+b\right)}{a(2 b+2+a)} .
$$

The last of these terms is positive; hence,

$$
\begin{aligned}
g(1+b, a) & \geq 2+\eta-\frac{2+\eta+(1-\eta) b}{2 b+2+a} \geq 2+\eta-\frac{2+1+b}{2 b+2+a} \\
& \geq 2+\eta-1=1+\eta=g(0,1) .
\end{aligned}
$$

This shows that $g(x, a) \geq g(0,1)=1+\eta$ for all $x, a$, and in particular, for the choices of $\gamma$ we allow, $\gamma \leq 1+\eta \leq g(x, a)$.

\section{PROOFS FOR SECTION 6}

Proposition 6.3. Let $(E, \boldsymbol{r}, \boldsymbol{d}, \alpha)$ be an instance with uniform altruism $\alpha \geq 0$. Then, $f$ is a Nash equilibrium for $\alpha$-altruistic players if and only if it minimizes $\sum_{S \subseteq E} d_{S}^{(\alpha)}(f) \tilde{f}_{S}$ over all solutions $\tilde{f}$ feasible for $(E, \boldsymbol{r})$.

Proof. By fixing a Nash equilibrium $f, \sum_{S} d_{S}^{(\alpha)}(f) \tilde{f}_{S}$ is the social cost of a feasible solution $\tilde{f}$, where the cost of each set $S$ is the congestion-independent constant $d_{S}^{(\alpha)}(f)$. If a flow $f$ is at Nash equilibrium for $\alpha$-altruistic players, then a player of type $\mathcal{S}$ selects a set $S \in \mathcal{S}$ to minimize $d_{S}^{(\alpha)}(f)$, so $\sum_{S} d_{S}^{(\alpha)}(f) \tilde{f}_{S}$ is minimized over all feasible solutions $\tilde{f}$. Conversely, if a solution $\tilde{f}$ is not at Nash equilibrium for $\alpha$-altruistic players, a player can decrease his (perceived) cost at $\tilde{f}$ by deviation. Thus, $\sum_{S} d_{S}^{(\alpha)}(f) \tilde{f}_{S}$ (or, equivalently, $\left.\sum_{e} d_{e}^{(\alpha)}\left(f_{e}\right) \tilde{f}_{e}\right)$ is not minimized.

THEOREM 6.7. If $(E, \boldsymbol{r}, \boldsymbol{d}, \alpha)$ has delay functions in $\mathcal{C}_{p}$, then the coarse price of anarchy is at most

$$
\left(\left(\frac{1+\alpha p}{1+p}\right)^{1 / p}\left(\frac{1+\alpha p}{1+p}-1-\alpha p\right)+1+\alpha p\right)^{-1}
$$


Proof. As observed in Roughgarden [2005], it suffices to focus only on polynomials $d(x)=a x^{i}$ with $x \leq p$. For any instance $(E, \boldsymbol{r}, \boldsymbol{d}, \alpha)$ with arbitrary polynomials can be equivalently transformed into one with only such monomials, by replacing each facility with delay function $d_{e}(x)=\sum_{i=0}^{p} a_{i} x^{i}$ by a set of $p+1$ new facilities, the $i^{\text {th }}$ of which has delay function $\tilde{d}_{e, i}(x)=a_{i} x^{i}$. In order to compute the anarchy value $\sigma(d)$ of a nonzero polynomial function $d(x)=a x^{i}$, we first write $x=\lambda r$ in Eq. (13) and cancel out a factor $r$, obtaining that

$$
\sigma^{(\alpha)}(d)=\sup _{r \geq 0} \max _{\lambda \geq 0}\left(\frac{\lambda d(\lambda r)}{d(r)}+(1-\lambda)\left(1+\frac{\alpha r d^{\prime}(r)}{d(r)}\right)\right)^{-1} .
$$

For any $r$, let $\lambda_{r} \geq 0$ be the maximizer of this expression. Setting the derivative of the expression to zero shows that $\lambda_{r}$ must satisfy $d^{(1)}\left(\lambda_{r}\right)=d^{(\alpha)}(r)$. Notice that because

$$
d^{(1)}(0 \cdot r) \leq d^{(\alpha)}(r) \leq d^{(1)}(r)
$$

and $d^{(1)}(\cdot)$ is continuous, there is in fact a $\lambda \in[0,1]$ solving the equation.

Next, we calculate $\lambda_{r}$ in the special case $d(x)=a x^{i}$. Solving the equation $d^{(1)}\left(\lambda_{r}\right)=$ $d^{(\alpha)}(r)$ for $\lambda_{r}$ gives us that $\lambda_{r}=\left(\frac{1+\alpha i}{1+i}\right)^{1 / i}$, which is independent of $r$. Thus,

$$
\frac{d\left(\lambda_{r} r\right)}{d(r)}=\frac{1+\alpha i}{1+i} \quad \text { and } \quad \frac{d^{\prime}(r)}{d(r)}=\frac{i}{r}
$$

Substituting these values into the expression for $\sigma^{(\alpha)}(d)$ gives us that

$$
\sigma^{(\alpha)}(d)=\left(\left(\frac{1+\alpha i}{1+i}\right)^{1 / i}\left(\frac{1+\alpha i}{1+i}-1-\alpha i\right)+1+\alpha i\right)^{-1}
$$

which is independent of $a$ and increasing in $i$ (by a derivative test). Hence, the largest $\sigma(d)$ is attained for $d=x^{p}$, giving

$$
\sigma^{(\alpha)}\left(\mathcal{C}_{p}\right)=\left(\left(\frac{1+\alpha p}{1+p}\right)^{1 / p}\left(\frac{1+\alpha p}{1+p}-1-\alpha p\right)+1+\alpha p\right)^{-1}
$$

as claimed.

\section{PROOF FOR SECTION 7}

Proposition 7.5. Conditioned on the mean of $\psi$ being any given $\bar{\alpha}$, the quantity $\left(\int_{0}^{1} \psi(t) \frac{1}{\sigma^{(t)}(\mathcal{C})} d t\right)^{-1}$ is maximized when $\psi$ has a point mass of $\bar{\alpha}$ on 1 and $1-\bar{\alpha}$ on 0 . It is minimized when $\psi$ has a point mass of 1 on $\bar{\alpha}$.

Proof. We will show that $\frac{1}{\sigma^{(\alpha)}(\mathcal{C})}$ is concave as a function of $\alpha$. Both results then follow readily from Jensen's Inequality. To prove concavity, let $p_{1}, p_{2} \geq 0$ satisfy $p_{1}+p_{2}=1$. For any delay function $d \in \mathcal{C}$, writing $x=\lambda r$ in Definition 6.5 and canceling a factor $r$ 
gives us

$$
\begin{aligned}
\frac{1}{\sigma^{\left(p_{1} \alpha_{1}+p_{2} \alpha_{2}\right)}(d)}= & \inf _{r, \lambda \geq 0} \frac{\lambda d(\lambda r)+(1-\lambda) d(r)+(1-\lambda)\left(p_{1} \alpha_{1}+p_{2} \alpha_{2}\right) d^{\prime}(r)}{d(r)} \\
= & \inf _{r, \lambda \geq 0}\left(\frac{p_{1}\left(\lambda d(\lambda r)+(1-\lambda) d(r)+(1-\lambda) \alpha_{1} d^{\prime}(r)\right)}{d(r)}\right. \\
& \left.\quad+\frac{p_{2}\left(\lambda d(\lambda r)+(1-\lambda) d(r)+(1-\lambda) \alpha_{2} d^{\prime}(r)\right)}{d(r)}\right) \\
\geq & \inf _{r, \lambda \geq 0} \frac{p_{1}\left(\lambda d(\lambda r)+(1-\lambda) d(r)+(1-\lambda) \alpha_{1} d^{\prime}(r)\right)}{d(r)} \\
& \quad+\inf _{r, \lambda \geq 0} \frac{p_{2}\left(\lambda d(\lambda r)+(1-\lambda) d(r)+(1-\lambda) \alpha_{2} d^{\prime}(r)\right)}{d(r)} \\
= & p_{1} \frac{1}{\sigma^{\left(\alpha_{1}\right)}(d)}+p_{2} \frac{1}{\sigma^{\left(\alpha_{2}\right)}(d)} .
\end{aligned}
$$

Finally, we take an infimum over all $d \in \mathcal{C}$ on both sides to complete the proof of concavity.

\section{REFERENCES}

H. Ackermann, H. Röglin, and B. Vöcking. 2006. Pure Nash equilibria in player-specific and weighted congestion games. In Proceedings of the 2nd Workshop on Internet and Network Economics.

A. Anagnostopoulos, L. Becchetti, B. De Keijzer, and G. Schäfer. 2013. Inefficiency of games with social context. In Proceedings of the 6th International Symposium on Algorithmic Game Theory.

E. Anshelevich, O. Bhardwaj, and M. Hoefer. 2012. Friendship, altruism, and reward sharing in stable matching and contribution games. http://arxiv.org/pdf/1204.5780.pdf.

E. Anshelevich, A. Dasgupta, J. Kleinberg, E. Tardos, T. Wexler, and T. Roughgarden. 2004. The price of stability for network design with fair cost allocation. In Proceedings of the 45th Symposium on Foundations of Computer Science.

V. Arya, N. Garg, R. Khandekar, A. Meyerson, K. Munagala, and V. Pandit. 2004. Local search heuristics for K-median and facility location problems. SIAM J. Comput. 33, 3.

R. J. Aumann. 1974. Subjectivity and correlation in randomized strategies. J. Math. Econ. 1, 1.

B. Awerbuch, Y. Azar, and A. Epstein. 2005. Large the price of routing unsplittable flow. In Proceedings of the 37th Annual ACM Symposium on Theory of Computing.

M. Babaioff, R. Kleinberg, and C. H. Papadimitriou. 2009. Congestion games with malicious players. Games Econ. Behav. 67, 1.

T. Bergstrom. 1999. Systems of benevolent utility functions. J. Public Econ. Theory 1, 1.

Vittorio Bilò, Alessandro Celi, Michele Flammini, and Vasco Gallotti. 2013. Social context congestion games. Theoret. Comput. Sci. 514, 21-35. (Graph Algorithms and Applications: in Honor of Professor Giorgio Ausiello.)

A. Blum, E. Even-Dar, and K. Ligett. 2006. Routing without regret: On convergence to Nash equilibria of regret-minimizing algorithms in routing games. In Proceedings of the 25th Annual ACM Symposium on Principles of Distributed Computing.

A. Blum, M. T. Hajiaghayi, K. Ligett, and A. Roth. 2008. Regret minimization and the price of total anarchy. In Proceedings of the 40th Annual ACM Symposium on Theory of Computing.

V. Bonifaci, T. Harks, and G. Schäfer. 2007. The impact of stackelberg routing in general networks. Tech. Rep. COGA Preprint 020-2007, TU Berlin.

M. Bradonjic, G. Ercal-Ozkaya, A. Meyerson, and A. Roytman. 2009. On the price of mediation. In Proceedings of the 11th ACM Conference on Electronic Commerce.

F. Brandt, T. Sandholm, and Y. Shoham. 2007. Spiteful bidding in sealed-bid auctions. In Proceedings of the 20th International Joint Conference on Artifical Intelligence.

R. Buehler, Z. Goldman, D. Liben-Nowell, Y. Pei, J. Quadri, A. Sharp, S. Taggart, T. Wexler, and K. Woods. 2011. The price of civil society. In Proceedings of the 7th International Workshop on Internet and Network Economics. 
I. Caragiannis, C. Kaklamanis, P. Kanellopoulos, M. Kyropoulou, and E. Papaioannou. 2010. The impact of altruism on the efficiency of atomic congestion games. In Proceedings of the 5th Symposium on Trustworthy Global Computing.

G. Charness and M. Rabin. 2002. Understanding social preferences with simple tests. Quart. J. Econ. 117.

P.-A. Chen, M. David, and D. Kempe. 2010. Better vaccination strategies for better people. In Proceedings of the 11th ACM Conference on Electronic Commerce.

P.-A. Chen, B. de Keijzer, D. Kempe, and G. Schäfer. 2011. The robust price of anarchy of altruistic games. In Proceedings of the 7th Workshop on Internet \& Network Economics.

P.-A. Chen and D. Kempe. 2008. Altruism, selfishness, and spite in traffic routing. In Proceedings of the 9th ACM Conference on Electronic Commerce.

X. Chen and X. Deng. 2006. Settling the complexity of two-player Nash-equilibrium. In Proceedings of the 47rd Symposium on Foundations of Computer Science.

G. Christodoulou and E. Koutsoupias. 2005. The price of anarchy of finite congestion games. In Proceedings of the 37th Annual ACM Symposium on Theory of Computing.

R. Cole, Y. Dodis, and T. Roughgarden. 2003. Pricing network edges for heterogeneous selfish users. In Proceedings of the 35th Annual ACM Symposium on Theory of Computing.

R. Cole, Y. Dodis, and T. Roughgarden. 2006. How much can taxes help selfish routing? J. Comput. System Sci. 72 .

S. Dafermos. 1972. The traffic assignment problem for multiclass-user transportation networks. Transport. Sci. 6.

J. Elias, F. Martignon, K. Avrachenkov, and G. Neglia. 2010. Socially-aware network design games. In Proceedings of the INFOCOM'10.

E. Fehr and K. M. Schmidt. 2005. The economics of fairness, reciprocity and altruism - Experimental evidence and new theories. Tech. Rep. Discussion Paper No. 2005-20, Department of Economics, Universität München.

A. Fiat, A. Karlin, E. Koutsoupias, and A. Vidali. 2013. Approaching utopia: Strong truthfulness and externality-resistant mechanisms. In Proceedings of the 4th Conference on Innovations in Theoretical Computer Science.

J. Geanakoplos, D. Pearce, and E. Stacchetti. 1989. Psychological games and sequential rationality. Games Econ. Behav. 1, 1.

H. Gintis, S. Bowles, R. Boyd, and E. Fehr. 2005. Moral Sentiments and Material Interests : The Foundations of Cooperation in Economic Life. MIT Press.

W. D. Hamilton. 1963. The evolution of altruistic behavior. Amer. Natural. 97.

W. D. Hamilton. 1964. The genetical evolution of social behavior, I\&II. J. Theoret. Biol. 7.

J. Hannan. 1957. Approximation to Bayes risk in repeated plays. Contrib. Theory Games 3.

A. Hayrapetyan, E. Tardos, and T. Wexler. 2006. The effect of collusion in congestion games. In Proceedings of the 38th Annual ACM Symposium on Theory of Computing.

M. Hoefer and A. Skopalik. 2009a. Altruism in atomic congestion games. In Proceedings of the 17th European Symposium on Algorithms.

M. Hoefer and A. Skopalik. 2009b. Stability and convergence in selfish scheduling with altruistic agents. In Proceedings of the 5th International Workshop on Internet and Network Economics.

P. Hui, K. Xu, V. O. K. Li, J. Crowcroft, V. Latora, and P. Lio. 2009a. Impact of altruism on opportunistic communications. In Proceedings of the 1st International Conference on Ubiquitous and Future Networks.

P. Hui, K. Xu, V. O. K. Li, J. Crowcroft, V. Latora, and P. Lio. 2009b. Selfishness, altruism and message spreading in mobile social networks. In Proceedings of the INFOCOM'09 Workshop.

G. Karakostas and A. Viglas. 2007. Equilibria for networks with malicious users. Math. Program. 110.

E. Koutsoupias and C. Papadimitriou. 1999. Worst-case equilibria. In Proceedings of the 16th Annual Symposium on Theoretical Aspects of Computer Science.

J. Ledyard. 1997. Public goods: A survey of experimental resesarch. In Handbook of Experimental Economics, J. Kagel and A. Roth (Eds.), Princeton University Press.

D. K. Levine. 1998. Modeling altruism and spitefulness in experiments. Rev. Econ. Dynam. 1, 3 (July 1998).

T. Lücking, M. Mavronicolas, B. Monien, and M. Rode. 2008. A new model for selfish routing. Theoret. Comput. Sci. 406 (October 2008). Issue 3.

A. Mas-Colell. 1984. On a theorem of Schmeidler. J. Math. Econ. 13.

D. Meier, Y. A. Oswald, S. Schmid, and R. Wattenhofer. 2008. On the windfall of friendship: Inoculation strategies on social networks. In Proceedings of the 10th ACM Conference on Electronic Commerce. 
I. Milchtaich. 1996. Congestion games with player-specific payoff functions. Games Econ. Behav. $13,1$.

I. Milchtaich. 2012. Comparative statics of altruism and spite. Games Econ. Behav. 75, 2.

T. Moscibroda, S. Schmid, and R. Wattenhofer. 2006. When selfish meets evil: Byzantine players in a virus inoculation game. In Proceedings of the 25th Annual ACM Symposium on Principles of Distributed Computing.

U. Nadav and T. Roughgarden. 2010. The limits of smoothness: A primal-dual framework for price of anarchy bounds. In Proceedings of the 6th Workshop on Internet and Network Economics.

N. Nisan, T. Roughgarden, É. Tardos, and V. V. Vazirani (Eds.). 2007. Algorithmic Game Theory. Cambridge University Press.

A. Pigou. 1920. The Economics of Welfare. Macmillan.

M. Rabin. 1993. Incorporating fairness into game theory and economics. Amer. Econ. Rev. 83, 5 (December 1993).

M. Rahn and G. Schäfer. 2013. Bounding the inefficiency of altruism through social contribution games. In Proceedings of the 9th Conference on Web and Internet Economics.

R. W. Rosenthal. 1973. A class of games possessing pure-strategy Nash equilibria. Int. J. Game Theory 2.

A. Roth. 2008. The price of malice in linear congestion games. In Proceedings of the 4th Workshop on Internet and Network Economics.

T. Roughgarden. 2004. Stackelberg scheduling strategies. SIAM J. Comput. 33.

T. Roughgarden. 2005. Selfish Routing and the Price of Anarchy. MIT Press.

T. Roughgarden. 2009. Intrinsic robustness of the price of anarchy. In Proceedings of the 41st Annual ACM Symposium on Theory of Computing.

T. Roughgarden and F. Schoppmann. 2011. Local smoothness and the price of anarchy in atomic splittable congestion games. In Proceedings of the 22nd ACM-SIAM Symposium on Discrete Algorithms.

T. Roughgarden and E. Tardos. 2000. How bad is selfish routing? In Proceedings of the 41st Symposium on Foundations of Computer Science.

M. Smith. 1979. The marginal cost taxation of a transportation network. Transport. Res. Part B, 13.

C. Swamy. 2007. The effectiveness of stackelberg strategies and tolls for network congestion games. In Proceedings of the 18th ACM-SIAM Symposium on Discrete Algorithms.

A. Vetta. 2002. Nash equilibria in competitive societies, with applications to facility location, traffic routing and auctions. In Proceedings of the 43rd Symposium on Foundations of Computer Science.

S. A. West, C. El Mouden, and A. Gardner. 2011. Sixteen common misconceptions about the evolution of cooperation in humans. Evolut. Hum. Behav. 32, 4.

H. P. Young. 1995. Strategic Learning and its Limits. Oxford University Press.

Received August 2013; revised March 2014; accepted May 2014 